\title{
A MODIFIED METHOD FOR THE. DETERMINATION OF RELATIVE WAVE-LENGTHS
}

\author{
By Irwin G. Priest
}

\section{CONTENTS}

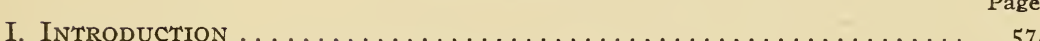

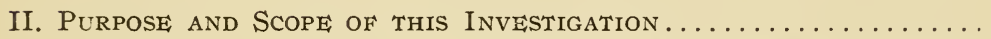

III. Fundamental Principles of Relative Wave-Length Determina-

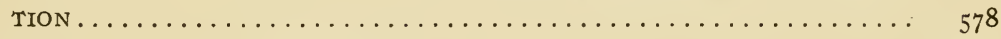

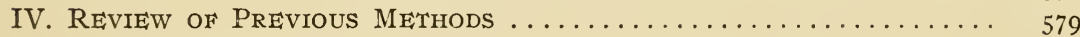

I. Earliest Method.............................. 579

2. Grating Methods ............................. 580

Simple dispersion method. The method of coincidences.

3. Interferometer Methods..................... $5_{82}$

Michelson's method. Fabry and Perot's method of coincidences. Fabry and Perot's method of diameters.

V. General Exposition of the Present Method .............. 586

I. Characteristic Fcatures . . . . . . . . . . . . . 586

Use of circular fringes in reflected light. Use of double increment in distance between mirrors as difference of path. Use of method of flexure to measure fractional part of order of interference.

2. Abstract Outline of Procedure.................. 588

Essential arrangements and conditions............ $\quad 5^{88}$

Determination of order of interference for standard wave-

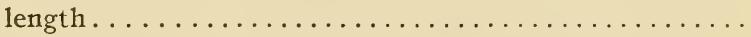

Determination of order of interference for unknown wave-

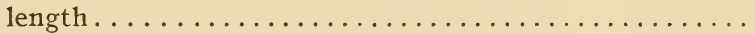

Reduction of required quantities to directly measured quantities.............................. 590

3. Summary of Requisite Data ................. $59^{\mathrm{I}}$

VI. The Apparatus and Its Arrangement ...................... 592

ViI. Experimental Details and Precautions . . . . . . . . . . . . . . 594

I. Preliminary Adjustments.................... 594

2. Course of a Single Determination............... 595

3. Special Precautions. ......................... 596

Vili. Computation ................................... 597 


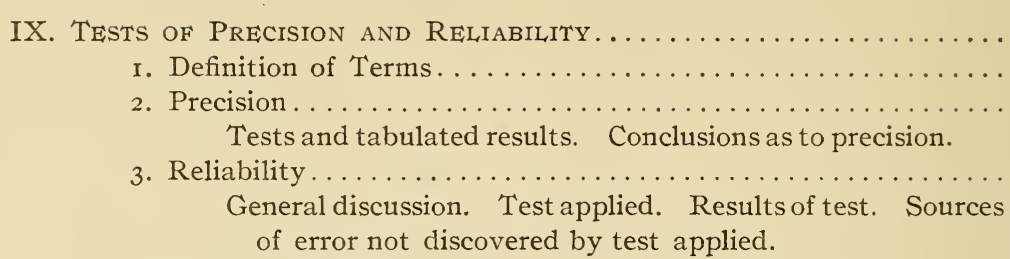

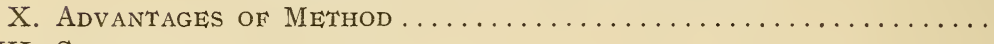

\section{INTRODUCTION}

For a proper understanding of the subject of this paper the reader should be familiar with the work that has been done in the past decade for the establishment of a new system of standard wave-lengths. ${ }^{1}$

An excellent résumé of the work of Michelson and of Fabry and Perot, as well as an exposition of the circumstances of the loss of confidence in the extreme accuracy of Rowland's system of spectroscopic standards, will be found in Chapter IX of Baly's Spectroscopy (Longmans, I905). The history of the organized effort that has been made since 1904 to establish an "International System of Wave-Length Normals" is recorded in the transactions of the International Union for Cooperation in Solar Research, Volumes I and II (Manchester, I906, I 908).

In sections II-IV of this paper is presented such theoretical and historical matter as the author considers requisite to form a logical introduction to his own work. A comprehensive historical résumé has not been attempted, and it is not expected that these sections will be sufficient introduction for the reader entirely unacquainted with the previous researches noted above; on the

1 The following are the important memoirs on this subject.

Michelson, Trav. et Mem. du Bur. Int., 11, p. 3; 1895.

Fabry and Perot, Ann. de Chim. et de Phys. (7), 16, p. 289; 1899.

Fabry and Perot, Ann. de Chim. et de Phys. (7), 25, p. 98; 1902.

(Trans. in Astrop. J., 15, pp. 73 and 261.)

Rayleigh, Phil. Mag. 11, p. 685, 1906, and 15, p. 548; 1908.

Buisson and Fabry, J. de Phys. (4), 7, p. I69; 1908. (Trans. in Astro. Jour. 28, p. I69.)

Evershein, Zs. wiss. Photo., 5, p. 152; 1907. (Trans. in Astro. Jour., 26, p. I72.) Ainn. der Physik, 30, p. 815; 1909.

Benoit, Fabry and Perot, C. R., 144, p. I082. Trans. Inter. Union Solar Res., 2, p. 109; 1908.

Pfund, Astro. Jour., 28, p. 197; 1908. 
other hand, III and IV will be of no interest to readers already familiar with such investigations. Section $V$ is intended to be sufficient in itself to give anyone already acquainted with this field of investigation a clear idea of the principles applied and the general procedure followed in the present method. Sections VI, VII, and VIII are taken up wholly in details of experiment and computation. They will only be of interest in case it is desired to examine the method critically or to duplicate the apparatus or procedure. Section IX presents the tests we have made of the precision and reliability of the method, and Section X summarizes the advantages that are claimed for it.

Schedule of Symbols-Symbols not defined in the immediate context where they appear should be understood as follows.

$\lambda$, wave-length.

$\lambda_{x}$, the unknown wave-length whose accurate value is sought.

$\lambda_{s}$, the standard reference wave-length, relative to which $\lambda_{x}$ is to be determined.

$\lambda_{x a}$, the approximate value of $\lambda_{x}$, known before the present determination.

$\lambda_{r}$, the auxiliary reference wave-length, i. e., a wave-length known with an accuracy about the same as that of $\lambda_{s}$ and used in the process of determining $\Delta P_{s e}$.

$t$, perpendicular distance between interferometer mirrors.

$d$, difference of path.

$d_{o}$, difference of path at center of circular fringe system $=($ subject to a small correction) $2 t$.

$n=\frac{d}{\lambda}$, order of interference.

$p=\frac{d_{o}}{\lambda}$, order of interference corresponding to center of circular fringe system.

$N$, integral part of $n$.

$\eta$, fractional part of $n$.

$P$, integral part of $p$.

$\phi$, fractional part ${ }^{2}$ of $p$.

${ }^{2} \phi$ as defined here is not rigorously equal to $\phi$ as found in the computing forms; but as $\phi$ only enters the computation in the form $\left(\phi_{2}-\phi_{1}\right)$ and the errors of these two may be assumed equal, no error will be produced in the result. It is therefore not thought necessary to introduce another symbol. 
$t_{1}$ and $t_{2}$, particular values of $t$ such that $t_{1}<t_{2}$.

$\Delta t=t_{2}-t_{1}$.

$\Delta p=\frac{2 \Delta t}{\lambda}$.

$\Delta P$, integral part of $\Delta p$.

$\Delta \phi$, fractional part of $\Delta p$.

Subscripts $I$ and 2 indicate that the symbols to which they are appended represent particular values for the conditions $t=t_{1}$ and $t=t_{2}$, respectively. Subscripts $s, r$, and $x$ indicate that the symbols to which they are appended represent the indicated quantities for the conditions $\lambda=\lambda_{s}, \lambda=\lambda_{r}$, and $\lambda=\lambda_{x}$, respectively.

$t_{1 a}$ indicates an approximate value of $t_{1}$, never entering our computation and only given as incidental datum.

$\Delta t_{a}$ indicates an approximate value of $\Delta t$, determined by $\Delta t_{a}=$ $M_{2}-M_{1}+K$ where $M_{2}$ and $M_{1}$ are micrometer-microscope readings, and $K$ is the scale correction.

$\Delta t_{e}$ indicates the accurate value of $\Delta t$ determined by $\Delta t_{e}=$ $\Delta P_{s e} \lambda_{s}$.

$\Delta P_{s a}$ indicates the approximate value of $\Delta P_{s}$ determined by $\Delta P_{s a}=\frac{2 \Delta t_{a}}{\lambda_{s}}$.

$\Delta P_{s e}$ indicates the exact value of $\Delta P_{s}$.

$\Delta p_{x a}$ indicates the approximate value of $\Delta p_{x}$ determined by $\Delta p_{x a}=\frac{2 \Delta t_{e}}{\lambda_{x a}}$.

$\Delta p_{x e}$ indicates the accurate value of $\Delta p_{x}$.

$\Delta \phi_{r m}$ indicates experimental value of $\Delta \phi_{r}$, determined by $\Delta \phi_{r m}=\phi_{r 2}\left(\right.$ or $\left.\mathrm{I}+\phi_{r_{2}}\right)-\phi_{r_{1}}$.

$\Delta \phi_{r c}$ indicates value of $\Delta \phi_{r}$, determined by $\Delta \phi_{r c}=$ fractional part of $\Delta P_{s e} \cdot \frac{\lambda_{s}}{\lambda_{r}}$.

$\AA$, Ångström unit.

$\AA_{M}, \frac{\lambda \text {, red cadmium radiation }}{6438.4722}$.

$\delta$ prefixed to the symbol for any quantity indicates a small error in that quantity.

$E$ indicates probable error as defined in the theory of errors. 
The quantity to which $E$ refers is indicated by writing its symbol as a subscript to $E$.

This work has been done in the laboratory of Dr. S. W. Stratton, to whom is due the design of the interferometer mounting and the flexure apparatus as well as general supervision of the work. The author has also had the privilege of consulting with Dr. A. H. Pfund, of the Johns Hopkins University, who has kindly reviewed and criticised this paper while in manuscript. Our hearty thanks are due him for his interest and service in this connection. Mr. C. F. Snyder has assisted in observing and computing.

\section{PURPOSE AND SCOPE OF THIS INVESTIGATION}

The purpose of this research has been. (I) to develop, along the lines indicated under $\mathrm{V}$, a method for the determination of relative wave-lengths, which would meet the present requirements for the establishment of secondary spectroscopic standards; and (2) to test the precision and reliability of this method.

Let us recall what the requirements are. The consensus of opinion among spectroscopists in regard to wave-length standards is: (I) that some one wave-length should be adopted as the primary standard; (2) that a large number of secondary standards distributed through the spectrum at intervals not greater than $50 \AA$ should be established relative to this primary by methods as direct and simple as possible; (3) that the uncertainty in the established values of these standards should not be greater than the uncertainty incident to a wave-length determination by the method of interpolation between neighboring standards in a grating spectrum. The accuracy of this method has heretofore been given ${ }^{3}$ as about $\pm 0.005 \AA$ or one part in I 000000 . More recently it has been asserted ${ }^{4}$ that, under the most favorable conditions, this uncertainty is not greater than about $\pm 0.001 \AA$ or, for the wave-lengths in question, one part in 4000 ooo. We may then take as our ideal of accuracy one part in 4000 ooo, mean-

${ }^{3}$ Kayser, Trans. Inter. Union Solar Research, 1, p. 39; I906 Fabry and Buisson, Trans. Inter. Union Solar Res., 2, p. ${ }_{3} 8$.

${ }^{4}$ St. John. Astro. Jour., 31, p. 156; March, I910. 
ing thereby that we desire the difference between the established and the true value to be certainly not greater than that amount. At the same time let us note that determinations having two or three times this uncertainty would still be of great value, although they should not be regarded as final so long as there is any possibility of improving them.

The method which I have developed, apparently satisfies the above requirements very closely and is believed to possess some peculiar advantages. The purpose of this paper is (I) to describe the method as it is now being used in this laboratory, and (2) to present the experimental evidence which has been obtained relative to its precision and reliability.

It will be convenient, first, to outline the principles upon which any determination of relative wave-length must be based.

\section{FUNDAMENTAL PRINCIPLES OF RELATIVE WAVE- LENGTH DETERMINATION}

Any comparison of two wave-lengths must, of necessity, be made by some interference method; and, on the other hand, the wave-length of any radiation relative to that of any other may be derived from suitable observations on some form of interference phenomenon. The fundamental equation of condition that must be used in evaluating one wave-length in terms of another is

$$
n_{x} \lambda_{x}=n_{s} \lambda_{s}
$$

The essential problem of a determination of relative wave-length is, then, the determination of two numbers, $n_{x}$ and $n_{s}$. In particular, the problem is: (I) to select some interference phenomenon yielding a measurable quantity or quantities of which $n$ is a function; (2) to set up some interferometer and auxiliary apparatus enabling these quantities to be measured when the interferometer is illuminated by lights of wave-lengths $\lambda_{x}$ and $\lambda_{s}$, and the condition, $n_{x} \lambda_{x}=n_{s} \lambda_{s}$, is satisfied; (3) to measure the quantities from which $n_{x}$ and $n_{s}$ can be evaluated; and (4) to derive $n_{x}$ and $n_{s}$ from the experimental data and evaluate $\lambda_{x}$ from

$$
\lambda_{x}=\frac{n_{s} \lambda_{s}}{n_{x}}
$$


In general, $n$ will not be exactly integral, but will be the sum of an integer and a proper fraction. The exigencies of experiment and discussion frequently make it convenient to speak of these two parts separately. So we define

$N$ is the integral part of $n$ and

$\eta$ is the fractional part of $n$

so that

$$
n=N+\eta \text {. }
$$

Since the relative error, $\frac{\delta \lambda}{\lambda}$, in the measured wave-length is directly proportional to $\frac{\delta \eta}{n}$, the relative error in $n$, there are obviously two methods by which $\frac{\delta \lambda}{\lambda}$ can be made small, viz: (I) $N$ may be chosen very large or (2) $\eta$ may be measured with great precision. The degree of homogeneity of the light sets a natural maximum limit to $N$, which limit is of the order of hundreds of thousands $\left(\mathrm{IO}^{5}\right)$ for even the most nearly homogeneous radiations. But long before this limit is reached the increase in $N$ becomes antagonistic to the precise measurement of $\eta$. In all methods now available, including the present, this limitation comes to be markedly felt in the region where $N$ is of the order $\mathrm{IO}^{4}$.

Variations in the procedure for obtaining $n_{x}$ and $n_{s}$ constitute the differences, more or less great, in methods of relative wavelength determination. Several of these methods that have been of great importance will now be mentioned.

\section{REVIEW OF PREVIOUS METHODS}

\section{EARLIEST METHOD}

It is merely of historic interest to recall that the earliest discovered phenomenon affording the requisite data for the determination of relative wave-lengths was "Newton's Rings" (I676). The precision attainable in this classic method is limited both by the low values of $N$ available and the comparatively great uncertainty in the measurement of $\eta$. A higher degree of precision than $0.1 \%$ would probably be unattainable adhering to this method in its simplicity. 


\section{GRATING METHODS}

While Young actually did compute from Newton's data not only the relative, but also the absolute wave-lengths for the several spectrum "colors," we can hardly class these values as standards of wave-length; and we may say that all spectroscopic standards established prior to Michelson's evaluation of three wave-lengths in terms of the meter, were derived from observations on diffraction spectra produced by the grating. Let us outline the fundamental principles of the grating methods, considering the grating as a particular type of interferometer. If we throw the grating data for the determination of relative wavelengths into the form of the fundamental equation (I) we have, in general, for any grating and any combination of wave-lengths and orders,

$$
n_{x} \lambda_{x}=\left[\frac{n_{s}^{\prime}\left(\sin i \pm \sin \theta_{n_{x}}\right)}{\left(\sin i \pm \sin \theta_{n_{s}^{\prime}}\right)}\right] \lambda_{s}
$$

where

$i$ is angle of incidence,

$\theta_{n_{x}}$ is angle of diffraction for the point at which the difference of path equals $n_{x} \lambda_{x}$

$\theta_{n^{\prime} s}$ is angle of diffraction for the point at which difference of path equals $n_{s}^{\prime} \lambda_{s}$, and

the expression in brackets equals $n_{s}$ expressed as a function of the experimental data.

To obtain the requisite data for the evaluation of $\lambda_{x}, n_{x}$ and $n_{s}^{\prime}$ are always chosen arbitrarily as integers and then the corresponding angular measurements (or their equivalents) are made. To indicate that $n_{x}$ and $n_{s}^{\prime}$ are integral we write $N_{x}$ and $N^{\prime}{ }_{s}$ hereafter. $N_{x}$ and $N^{\prime}{ }_{s}$ are never greater than a few units and are obtained by direct count.

There are two special cases of importance. The first is distinguished by the condition

$$
N_{x}=N^{\prime}{ }_{s}
$$

all the observations being on the same "order" of spectrum, so that $\eta_{s}$ may have any value between $\mathrm{O}$ and $\mathrm{r}$. According to Kayser ${ }^{5}$ this method, using plane gratings, would have an accuracy 
of about 0.I $\bar{A}$. In practice it is replaced by the mcthod of linear interpolation between known standards.

The second is distinguished by the condition

$$
N_{x} \lambda_{x} \text { is very nearly equal to } N^{\prime}{ }_{s} \lambda_{s}
$$

so that $\eta_{s}$ is either very nearly o or very nearly I. Unless $\lambda_{x}$ is nearly equal to $\lambda_{s}$, this condition requires the condition

$$
N_{x} \neq N^{\prime}{ }_{s}
$$

so that observations are made on spectra of different orders. This is the well-known "method of coincidences." With plane gratings it is of small importance because it is embarrassed by the chromatism of the auxiliary lenses, but with the concave grating in Rowland's hands, it afforded the means of establishing his great system of spectroscopic standards. In Rowland's experimental method both $\theta_{N_{x}}$ and $\theta_{N^{\prime} s}$ were made either equal to zero or very nearly so. If

$$
\theta_{N_{x}}=\theta_{N^{\prime} s}=0
$$

we have simply $n_{s}=N_{s}^{\prime}$ and

$$
N_{x} \lambda_{x}=N_{s}^{\prime} \lambda_{s}
$$

which is the ordinary text-book form of presenting this method. However, the method would be of very limited application if only wave-lengths exactly satisfying (II) could be compared by it. Actually, Rowland obtained the small departure of $n_{s}$ from $N^{\prime}{ }_{s}$ (i. e., $\eta_{s}$ or $\mathrm{I}-\eta_{s}$ ) not from angular measurements as indicated by (6), indeed, but from linear measurements in the normal spectrum. We have only presented the method from this point of view in order to emphasize its fundamental relation to the methods which are commonly distinguished as "interference methods." " ${ }^{6}$ To summarize, we may say that Rowland's method, the first important method that we have to consider, is distinguished, regarding it from our present point of view, by: (I) the choice of an integral value for $n_{x}$; (2) the choice of very small unequal values for $N_{x}$ and $N_{s}$ readily obtained by count without any other artifice; (3) the choice of a very small value for $\eta_{s}$; (4) extreme accuracy of measurement of $\eta_{s}$ (about o.000 oor). 
Rowland's method has been most thoroughly examined and discussed in recent years. It is now universally admitted that the actual errors in his relative values, due to causes not recognized when it was published, may amount to several hundredths of an Ångström unit, although they were originally thought to be accurate to $0.01 \AA$. The inaccuracies of Rowland's method of coincidences were due to errors in the ruling of the grating. The essential defect of the method is thus the great mechanical difficulty of making a sufficiently good grating. A great advance was made by adopting radically different and much simpler apparatus. This simpler instrument, having much less serious intrinsic defects, is known as the interferometer. The two important forms have been the Michelson-Morley and the Fabry-Perot.

\section{INTERFEROMETER METHODS}

Incident to his absolute determination of the wave-lengths of three cadmium lines, Michelson obtained the relative values of these wave-lengths, and it is noteworthy that while the accuracy claimed for the absolute values was only about one part in 2000000 , the relative values are thought to be correct to about one part in $20000000 .^{7}$ The method of Michelson thus presents a possible means of determining relative wave-lengths. But Michelson's interferometer is subject to a limitation which would make its use in the establishment of an extensive system of standards quite inconvenient. This limitation is that the accuracy of measurement of $\eta$ is only about one part in ro. This means that to obtain an accuracy of one part in 4000 ooo in $\lambda, N$ would have to be 400000 , a value quite impracticable. In his work on the meter Michelson overcame this difficulty by his method of "stepping-up" from one difference of path to another It is the inconvenience of this process which would make the method unsuitable for an extensive research on a large number of lines. It has been found better to obtain the requisite accuracy in $\lambda$ by making the measurement of $\eta$ more accurate.

The more accurate measurement of $\eta$ with simple apparatus was made possible by Fabry and Perot's invention of an interferometer consisting of two parallel transparent mirrors of high reflecting power.

\footnotetext{
${ }^{7}$ Light Waves and their Uses, p. I04.
} 
Fabry and Perot's first method, known as their method of coincidences, is similar in fundamental principle to Rowland's method of the same name in that

$$
\eta_{x}=\eta_{s}=\mathrm{o}
$$

This first method did not make full use of the property of the new instrument in the accurate measurement of $\eta$. The error in $\eta$ may be as large as $0.05 .{ }^{8}$ To obtain the required accuracy in $n$ Fabry and Perot sought coincidences among spectra (fringes) of very high order $\left(N=10^{5}\right)$. They found it necessary to devise a very elaborate procedure to determine the integers $N_{x}$ and $N_{s}$; and although they determined a few relative wave-lengths by this method, they decided it was not entirely satisfactory for general application and soon abandoned it in favor of their second method or method of diameters.

Fabry and Perot's second method makes use of the same interference phenomenon as their first method, and, in so far as the optical parts are concerned, the interference apparatus in the two methods is identical. The radical difference between the two methods is this: In the first method $\eta_{x}$ and $\eta_{s}$ are each made equal to o by varying $t$ until this condition is satisfied. In the second method $t$ is constant and $\eta_{x}$ and $\eta_{s}$, which may have any values between $\mathrm{O}$ and $\mathrm{I}$, are evaluated from measurements on the interference fringes. The accidental uncertainty in $\eta$ is probably about $0.0 \mathrm{I}$ and $N$ is of the order $1 \mathrm{O}^{4}$. The still outstanding discrepancies between different investigators using this method vary between less than one part in 4000 ooo to more than one part in I 000 ooo for different lines and average roughly about one part in 2500000 . There is some reason for suspecting small systematic differences between different investigators, and there remain outstanding very serious discrepancies between Eversheim's recent determinations by this method and previously unquestioned determinations by other methods. ${ }^{9}$

\footnotetext{
${ }^{8}$ Ann. de Chim. et de Phys. (7), 16, p. 317; 1899.

${ }^{9}$ [Note added, November, IgIo.] See Priest, Certain Peculiarities of the Discrepancies among Recent Wave-Length Determinations. Communication to the American Physical Society, New York Meeting, October, Igro. Phy. Rev., November, IgIo. While this article has been going through the press, results have been published by Kayser showing that the accuracy of grating determinations has not yet been attained in the standards established by the method of diameters. See Astroph., J., 32, p. 2 I7; October, I9IO.
} 
As this is the only method in the field to-day, and so is the one with which our own method will naturally be compared, it is desirable to present it in a little more detail than the others. We shall therefore give a brief exposition of the essential features of this method as now practiced, not, however, taking space to justify the procedure followed. Also it will not be convenient or necessary to follow in detail the actual computation forms used; but the equations we shall give will exhibit the fundamental relations between the measured and computed quantities, and we will then summarize the nature and number of the measured quantities.

The interferometer used in this method consists of two plane, transparent, metallic mirrors supported on glass plates and accurately parallel to each other at a constant distance $(t)$ apart, this distance being maintained by the supporting action of small pieces of metal or alloy against which the glass plates are pressed by suitable springs. Light which has traversed the interferometer and suffered multiple reflections within it falls upon a convergent lens or mirror, in the principal focal plane of which is formed an interference figure consisting of alternately bright and dark concentric circles. The difference of path on which the computation is based is that giving rise to the interference at the center of this figure, and, to a first approximation, is equal to $2 t$. The required condition, $n_{x} \lambda_{x}=n_{s} \lambda_{s}(\mathrm{I})$, is then nearly satisfied by the approximate equation

$$
p_{x} \lambda_{x}=2 t=p_{s} \lambda_{s}
$$

The fundamental relation used to evaluate $p$ is the theoretically established equation

$$
p=\frac{n}{\cos i_{n}}
$$

or, to a sufficient approximation

$$
p=n\left(\mathrm{I}+\frac{i_{\mathrm{n}}^{2}}{2}\right)
$$

where $n$ is the order of interference of the bright ring which is the locus of image points for beams of parallel rays incident, on the interferometer mirrors, at the angle $i_{n}$. The exact integer $n$ may 
be determined by artifices which give its value without experimental error. Consequently the method of its determination need not be discussed here. The evaluation of $i_{n}$ is based upon the theoretically derived relation

$$
2 i_{n}=\alpha_{n}
$$

where $\alpha_{n}$ is the angle subtended at the second principal point of the lens (or the pole of the mirror) by $D_{n}$, the linear diameter of the ring of order $n$.

Three general methods have been used to determine $\alpha_{n}$. Fabry and Perot measured this angle by means of a telescope previously calibrated in terms of angle. Lord Rayleigh obtained it by measuring the angle through which the interferometer was turned to pass the distance $D_{n}$ past a fixed reference mark in the focal plane. Fabry and Buisson, Pfund, and Eversheim have evaluated $\alpha_{n_{\mathrm{s}}}$ and $\alpha_{n_{\mathrm{x}}}$ by means of the relations

$$
\begin{gathered}
\alpha_{n_{s}}=\frac{D_{n_{s}}}{F_{\lambda_{s}}}=\frac{\frac{h}{h_{\lambda_{s}}^{\prime}} D^{\prime}{ }_{n_{s}}}{F_{\lambda_{s}}}=\frac{\theta_{\lambda_{s}} D_{n_{3}}^{\prime}}{h_{\lambda_{s}}^{\prime}} \\
\alpha_{n_{x}}=\frac{D_{n_{x}}}{F_{\lambda_{x}}}=\frac{\frac{h}{h_{\lambda_{x}}^{\prime}} D^{\prime}{ }_{n_{x}}}{F_{\lambda_{x}}}=\frac{\theta_{\lambda_{s}} F_{\lambda_{s}} D_{n_{x}}^{\prime}}{h_{\lambda_{x}}^{\prime} F_{\lambda_{x}}}
\end{gathered}
$$

where the newly introduced symbols have significance as follows.

$h$, distance between two marks on a line coincident in direction and position with $D_{n_{s}}$ and $D_{n_{x}}$.

$F_{\lambda_{s}}$ and $F_{\lambda_{x}}$, focal lengths of the objective for light of wavelengths $\lambda_{s}$ and $\lambda_{x}$, respectively.

$\theta_{\lambda_{s}}$, angle subtended by $h$ as viewed through the objective when illuminated by light of wave-length $\lambda_{s}$.

$D^{\prime}{ }_{n_{s}}$ and $D^{\prime}{ }_{n_{x}}$, the negative photographic images of $D_{n_{3}}$ and $D_{n_{x}}$ formed by the action of an optical train containing convergent and dispersive elements.

$h^{\prime}{ }_{\lambda_{s}}$ and $h_{\lambda_{x}}^{\prime}$, the negative photographic images of $h$ formed, respectively, by light of wave-lengths $\lambda_{s}$ and $\lambda_{x}$ through the action of the just-mentioned train. The quantities experimentally measured were: by Fabry and Buisson, $\theta_{\lambda_{3}}, h_{\lambda_{s}}^{\prime}, h_{\lambda_{x}}^{\prime}, D_{n_{3}}^{\prime}, D_{n_{x}}^{\prime}$, $F_{\lambda_{s}}$ and $F_{\lambda_{x}}$; by Pfund and Eversheim, $h, h_{\lambda_{3}}^{\prime}, h_{\lambda_{x}}^{\prime}, D_{n_{s}}^{\prime}, D_{n_{x}}^{\prime}, F_{\lambda_{s}}$ 
and $F_{\lambda_{x}}$. However, with Pfund, $F_{\lambda_{x}}=F_{\lambda_{s}}$ rigorously, there being no chromatic aberration in his system; and Eversheim, in the examples he gives, ${ }^{10}$ puts $F_{\lambda_{x}}=F_{\lambda_{s}}$, thus implying that in the particular case he gives the aberration is negligible.

In addition to the foregoing, data must be obtained for evaluating the correction for the error due to "dispersion of phase" on reflection. The procedure for obtaining this data involves essentially another determination of the quantities $D^{\prime}{ }_{n_{s}}, D^{\prime}{ }_{n_{x}}, h_{\lambda_{s}}^{\prime}$, and $h^{\prime}{ }_{\lambda_{x}}$ for another value of $t$, or an operation about equivalent to this in number and character of observations.

Let us now examine the nature of these several quantities, reducing each to actual scale readings in so far as we can. $D^{\prime}{ }_{n_{s}}$, $D^{\prime}{ }_{n x}, h_{\lambda_{s}}^{\prime}, h^{\prime}{ }_{\lambda x}$, and $h$ are simple lengths, each obtained as the difference of two scale readings. $\theta_{\lambda_{s}}$ is an angle measured by a telescope with micrometer ocular. It is the difference of two scale readings. All of the foregoing quantities depend upon the previous accurate calibration of micrometer screws. None of the authors gives in any detail his method for the determination of $F_{\lambda_{x}}$ and $F_{\lambda_{s}}$, so that we are not in a position to reduce these to original scale readings.

Taking into account the observations necessary to make correction for "dispersion of phase," each wave-length determination is dependent upon I4 or I 8 scale readings plus whatever readings are involved in the determination of focal length. This enumeration of the number of readings does not count any repeated readings or analogous readings on different rings.

The essential differences in procedure between this method and our own will be made evident in the next section.

\section{GENERAL EXPOSITION OF THE PRESENT METHOD ${ }^{11}$}

\section{CHARACTERISTIC FEATURES}

The present method is characterized by three principal features.

The first characteristic is the use of the reflection fringes, the interferometer consisting of two parallel mirrors, one of which is

${ }^{10}$ Ann. d. Physik, 30, p. 836.

${ }^{11}$ This section should be considered in connection with the fundamental principles outlined under III above, the present method being a particular case of the general method there outlined. 
heavily silvered, while the other is silvered to a transmission of about so per cent, the light entering and leaving the interferometer through this partial mirror and in direction perpendicular to it. ${ }^{12}$ The silver mirrors are supported on plane glass plates. Light leaving the interometer falls upon a very short focus converging lens $(F=20 \mathrm{~mm})$, and the interference pattern of bright concentric rings is formed in the principal focal plane of this lens. The wideopen slit of a spectrometer is also placed in this focal plane and along a diameter of the rings. Observations are made at the spectrometer telescope. For each wave-length the observer sees an image of the spectrometer slit like a bright ribbon and in each ribbon the system of fringes due to that wave-length. As the difference of path is decreased the rings contract to bright spots and disappear one after the other at the center of the ring system, the difference of path being decreased by one wave-length between two successive disappearances. As will presently be shown, the measurement of the order of interference in this method depends essentially upon locating the relative positions of one interferometer mirror for the respective disappearances of certain bright spots in the fringe systems for the standard and the unknown wave-lengths. The precision with which these positions can be located limits the precision attainable in the measured wavelength. Hence the importance of being able to locate these positions precisely. The two essentials for attaining this end in the present method are the use of the reflection fringes and the short focus lens to form their image on the spectrometer slit. With this arrangement the observer can adjust the position of the mirror to the disappearance of the bright spot with great satisfaction and nicety. Regarding the quantitative measure of the precision of this adjustment I shall speak further on.

The second characteristic of the method is the use of the double increment in the distance between the two mirrors instead of the double distance as the difference of path.

The third characteristic is the use of the method of flexure to measure the fractional part of the order of interference. Use of the flexure of elastic material is, of course, not new in interferometry. It has been widely used in making fine adjustments,

\footnotetext{
${ }^{12}$ Hamy, Jour. de Phy. (4) 5, pp. 789-809; 1906. Priest, This Bulletin, 5, pp. 483-484; 1909 .
}

$48848^{\circ}-10-$ II 
and Michelson used torsional flexure to measure the "fractions" by means of his compensator. Fabry and Perot made use of minute flexures to adjust the mirrors of their interferometer, but, so far as I know, did not make measurements in that way. The general principle of the method as used in our apparatus is this: A lever, a weak spring, and a strong spring are so arranged that a displacement of 7500 of one end of the lever produces a displacement of about I of the interferometer mirror. We obtain by trial the displacement of the lever end necessary to produce a change of one wave-length in the difference of path. Assuming ${ }^{13}$ the apparatus to be obeying Hooke's law we can then calculate the increment of the difference of path produced by any measured displacement of the lever end. In this way fractional parts of the order of interference can be measured. ${ }^{14}$

\section{ABSTRACT OUTLINE OF PROCEDURE}

\section{ESSENTIAL ARRANGEMENTS AND CONDITIONS}

In Fig. I let $y_{1}$ be the trace of the heavily silvered mirror, its plane being perpendicular to the plane of the figure. Let $a_{1}, b_{1}$, $c_{1}, d_{1}$ be traces of the partial mirror in positions respectively as follows:
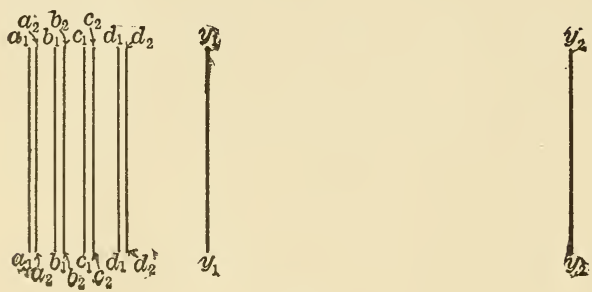

Note on Fig. I.-Not to scale. Relative positions of $a_{1}$ and $a_{2}, b_{1}$ and $b_{2}, c_{1}$ and $c_{2}, d_{1}$ and $d_{2}$ as to right and left must not be understood as determined by the figure, the purpose of which is merely to illustrate the indicated order $a, b, c, d, y_{1}, y_{2}$.

Fig. 1

$a_{1}$ is the position for disappearance of a bright spot in the fringe system for $\lambda_{s}$.

$d_{1}$ is the position for disappearance of the bright spot of next lower order in the same fringe system.

${ }^{13}$ The assumption that the large displacement of the lever end is proportional to the small displacement of the mirror can easily be tested experimentally by measuring the large displacements corresponding to successive increases of $I \lambda$ in the difference of path, and I have of ten done this.

${ }^{14}$ For the actual procedure followed, see VII, 2 and 3; and VIII below, where it is shown also that the effect of small departures from Hooke's law can be eliminated. 
$b_{1}$ is the position for first disappearance of a bright spot in system for $\lambda_{x}$ after leaving position $a_{1}$.

$c_{1}$ is the position for first disappearance of a bright spot in the system for $\lambda_{r}$, after leaving position $a_{1}$.

Let $y_{2}$ be trace of the heavily silvered mirror in another position, parallel to its first position and such that

$$
\overline{a_{1} y_{2}}>\overline{a_{1} y_{1}}
$$

For this position of the heavily silvered mirror, let $a_{2}, b_{2}, c_{2}, d_{2}$ have significance analogous to $a_{1}, b_{1}, c_{1}, d_{1}$, for the first position.

The equation we use for evaluating $\lambda_{x}$ in terms of $\lambda_{s}$ is

$$
\left(\Delta p_{x}\right) \lambda_{x}=2\left(\overline{a_{2} y_{2}}-\overline{a_{1} y_{1}}\right)=2 \Delta t=\left(\Delta p_{s}\right) \lambda_{s}
$$

We require the numerical values of $\Delta p_{s}$ and $\Delta p_{x}$

\section{DETERMINATION OF $\Delta p s$}

$\Delta p_{s}$ is determined by the following conditions.

$$
\begin{aligned}
\Delta p_{s} & =\text { an integer (by conditions of experiment). } \\
\Delta p_{s} & =\frac{2 \Delta t}{\lambda_{s}} \\
\left(\Delta p_{s}\right) \frac{\lambda_{s}}{\lambda_{r}} & =\text { some integer }+\Delta \phi_{r}
\end{aligned}
$$

For a given measured value of $\Delta t, \Delta t_{a}$, conditions (20) and (2 I) determine that $\Delta p_{s}$ must be an integer within the group of minor limit $L$ and major limit $L+l$, where $L$ is determined from $\lambda_{s}$ and $\Delta t_{a}$ while $l$ is determined by the accuracy of $\Delta t_{a}$. For a given measured value of $\Delta \phi_{r}$, say $\Delta \phi_{r m}$, conditions (20) and (22) determine that $\Delta p_{s}$ must be an integer in an arithmetic series of which the common difference, $j$, is determined by the ratio $\frac{\lambda_{s}}{\lambda_{r}}$. To determine $\Delta p_{s e}$ we reauire then measured values of $\Delta t$ and $\Delta \phi_{r}$, conditions being arranged to give $l$ and $j$ such values that only one member of the arithmetic series can fall within the group $L$ to $L+l . \Delta p_{s}$ is then determined uniquely as the integer within the group $L$ to $L+l$ satisfying (22) for $\Delta \phi_{r}=\Delta \phi_{r m}$. 


\section{DETERMINATION OF $\Delta p x$}

$\Delta p_{x}$ is determined by the following conditions.

$$
\begin{gathered}
\Delta p_{x}=\frac{2 \Delta t_{e}}{\lambda_{x}}=\frac{\Delta p_{s e} \lambda_{s}}{\lambda_{x}} \\
\Delta p_{x}=\text { an integer }+\Delta \phi_{x}
\end{gathered}
$$

Substituting the known values of $\Delta p_{s e}$ and $\lambda_{s}$, and $\lambda_{x a}$ the previously known approximate value of $\lambda_{x}$, condition (23) determines $\Delta p_{x}$ to be within a range of values the limits of which are fixed by the uncertainty in $\lambda_{x a}$. Substituting for $\Delta \phi_{x}$ its measured value, conditions (23) and (24) then determine $\Delta p_{x e}$, the accurate value of $\Delta p_{x}$, uniquely, provided that the uncertainty in $\lambda_{x a}$ was such as to make the uncertainty in $\Delta p_{x a}[$ from solution of (23)] numerically less than 0.5. [In symbols, $\left.+0.5>\frac{\left(\Delta p_{x}\right) \delta \lambda_{x}}{\lambda_{x}}>-0.5\right]$

To determine $\Delta p_{x e}$, we require then the values of $\Delta p_{s e}$ and $\lambda_{s}$, the measured value of $\Delta \phi_{x}$, and $\lambda_{x a}$, an approximate value of $\lambda_{x}$, with maximum uncertainty $\delta \lambda_{x}$, such that

$$
+0.5 \frac{\lambda_{x}}{\Delta p_{x}}>\delta \lambda_{x}>-0.5 \frac{\lambda_{x}}{\Delta p_{x}}
$$

The magnitudes to be obtained experimentally are then $\Delta t_{a}, \Delta \phi_{r}$ and $\Delta \phi_{x}$.

\section{REDUCTION OF REQUIRED QUANTITIES TO DIRECTLY MEASURED QUANTITIES}

The measurement of $\Delta t_{a}$ is a direct measurement obtained as the difference of two scale readings. A scale carried by the carriage carrying the movable mirror is read by a fixed micrometermicroscope before and after the displacement.

$\Delta \phi_{r}$ and $\Delta \phi_{x}$ are evaluated by means of the following equations,

$$
\Delta \phi_{r}=\frac{\overline{a_{2} c_{2}}}{\overline{a_{2} d_{2}}} \cdot \frac{\lambda_{s}}{\lambda_{r}}-\frac{\overline{a_{1} c_{1}}}{\overline{a_{1} d_{1}}} \cdot \frac{\lambda_{s}}{\lambda_{r}}
$$

and

$$
\Delta \phi_{x}=\frac{\overline{a_{2} b_{2}}}{\overline{a_{2} d_{2}}} \cdot \frac{\lambda_{s}}{\lambda_{x}}-\frac{\overline{a_{1} b}}{\overline{a_{1} d_{1}}} \cdot \frac{\lambda_{s}}{\lambda_{x}}
$$

the truth of which is evident from the construction given above. 
Inasmuch as conditions of measurement make the uncertainty in $\Delta \phi_{r}$ and $\Delta \phi_{x}$ greater than one part in I00o, there will always be at hand in advance sufficiently accurate values of $\lambda_{s}, \lambda_{r}$, and $\lambda_{x}$ for giving the ratios $\frac{\lambda_{s}}{\lambda_{r}}$ and $\frac{\lambda_{s}}{\lambda_{x}}$. The ratios $\frac{\overline{a_{1} c_{1}}}{{\overline{a_{1} d_{1}}}_{1}}, \frac{\overline{a_{1} b_{1}}}{\bar{a}_{1} d_{1}}, \frac{\overline{a_{2} c_{2}}}{\bar{a}_{2} d_{2}}, \frac{\overline{a_{2} b_{2}}}{\bar{a}_{2} d_{2}}$, are obtained by the direct measurement of six lengths $\overline{A_{1} C_{1}}, \overline{A_{1} B_{1}}$, $\overline{A_{1} D_{1}}, \overline{A_{2} C_{2}}, \overline{A_{2} B_{2}},{\overline{A_{2}}}_{2}$, directly proportional respectively to $\overline{a_{1} c_{1}}, \overline{a_{1} b_{1}}, \overline{a_{1} d_{1}}, \overline{a_{2} c_{2}}, \overline{a_{2} b_{2}},{\overline{a_{2}}}_{2}$, so that

$$
\begin{aligned}
& \frac{\overline{a_{1} c_{1}}}{\bar{a}_{1} d_{1}}=\frac{\overline{A_{1} C_{1}}}{\overline{A_{1} D_{1}}}, \frac{\overline{a_{1} b_{1}}}{\overline{a_{1} d_{1}}}=\frac{\overline{A_{1} B_{1}}}{\overline{A_{1} D_{1}}} \\
& \frac{\overline{a_{2} c_{2}}}{\overline{a_{2} d_{2}}}=\frac{\overline{A_{2} C_{2}}}{\overline{A_{2} D_{2}}} \text { and } \overline{\frac{a_{2} b_{2}}{\overline{a_{2} d_{2}}}}=\frac{\overline{A_{2} B_{2}}}{\overline{A_{2} D_{2}}}
\end{aligned}
$$

The lengths $\overline{A_{1} C_{1}}, \overline{A_{1} B_{1}}, \overline{A_{1} D_{1}}, \overline{A_{2} C_{2}}, \overline{A_{2} B_{2}}, \overline{A_{2} D_{2}}$, are measured in terms of the revolutions of a micrometer screw, the point of which bears on one end of a lever, the other end of which is attached by means of a weak spiral spring to a strong flat spring carrying the partial mirror, the strengths of the springs being adjusted so as to give the proportionality factor, 7500 , mentioned above.

\section{SUMMARY OF REQUISITE DATA}

The following is a list of the data requisite for the determination of a wave-length.

$A$. Reference standards and previously known values.

The standard reference wave-length, $\lambda_{s}$, and the auxiliary reference wave-length, $\lambda_{r}$

An approximate value of $\lambda_{x}, \lambda_{x a}$, such that

$$
+0.5 \frac{\lambda_{x}}{\Delta p_{x}}>\delta \lambda_{x}>-0.5 \frac{\lambda_{x}}{\Delta p_{x}}
$$

Correction $(K)$ of the scale used on the mirror carriage. $B$. Experimental data.

The displacement of the heavily silvered mirror in terms of scale divisions of the scale on the carriage.

The six lengths $\overline{A_{1} C_{1}}, \overline{A_{1} B_{1}}, \overline{A_{1} D_{1}}, \overline{A_{2} C_{2}}, \overline{A_{2} B_{2}}, \overline{A_{2} D_{2}}$, in terms of revolutions of the micrometer screw.

Temperature of the air and the scale on the mirror carriage. 
Atmospheric pressure. (Atmospheric temperature and pressure do not ordinarily enter the computation, and are only recorded to supply complete record in case of future question.)

The experimental data reduce to twelve scale readings, viz:

(I) $M_{1}, M_{2}$, micrometer-microscope readings on the scale before and after displacement of the carriage; (2) $A_{1}, B_{1}, C_{1}, D_{1}, A_{2}, B_{2}$, $C_{2}, D_{2}$, micrometer head readings; (3) thermometer reading; (4) barometer reading.

As to the way these several scale readings enter the calculation the following facts should be noticed.

Under ordinary laboratory conditions the temperature and pressure of the air influence the relative values so slightly as not to come into consideration at all.

$M_{1}, M_{2}, C_{1}$, and $C_{2}$, and the temperature of the scale are used only in determining the integral part of the order of interference, and as checks upon the reliability of the determination.

The precision of the measured wave-length is a function only of the accidental errors of $A_{1}, B_{1}, D_{1}, A_{2}, B_{2}, D_{2}$, and can be made to be sensibly a function of only the errors of $A_{1}, B_{1}, A_{2}, B_{2}$.

\section{THE APPARATUS AND ITS ARRANGEMENT}

The apparatus requisite for practicing the method consists of -

An interferometer, the essential elements and accessory parts of which are shown in Figs. 2 and 3 and enumerated in the key accompanying those figures; and

Accessory apparatus for illumination and observation arranged as shown in Fig. 4 and enumerated in the accompanying key.

The following is a list of the important special parts of this apparatus actually used in this laboratory.

Glass plates (I, 2, Fig. 2), by Pettididier, Chicago.

Interferometer bed, mirror mountings, and flexure apparatus, designed

by Dr. S. W. Stratton and constructed by Mr. O. G. Lange in the Bureau of Standards shop.

Micrometer screw (I I, Fig. 2), by Brown and Sharpe, $0.5 \mathrm{~mm}$ pitch, reading millimeters with smallest divisions on head indicating $0.01 \mathrm{~mm}$.

Scale (17, Fig. 2), $5 \mathrm{~cm}$ long, on silver, by Zeiss. Smallest division $=0.1 \mathrm{~mm}$. 
1,2 . Glass mirror plates, $x$ bearing the partial mirror and bearing the full mirror, which form the bounding surface surfaces beaing the siver tmirrors should be plane to, say, $\frac{\lambda}{10}$, the requirement in this respect not being severe. The second surface of plate 2 is, of course, a matter of indifference. The unsilvered surface of $\mathrm{I}$ should be as plane as good plate glass. The two surfaces of this plate should not be parallel, but may inclose an angle of about $2^{\text {? }}$.

3, 4 . Frames holding the mirror plates. $3 \mathrm{a}, 3 \mathrm{~b}, 3 \mathrm{c}$, clips to hold mirror plate in place. The clips are shown in the position for inserting and removing the mirror. (Similar clips on 4 not shown.

5a. Flat spring supporting 3 and being a part, in one piece, of 5

7. Spiral spring of piano wire connecting 6 and 8 .
8. Eyelet in 9 .

9. Lever pivoted at $\mathrm{roa}, \mathrm{Iob}$

ix. Micrometer screw bearing on 9 at $\mathrm{I}_{2}$.

(Continued below.)
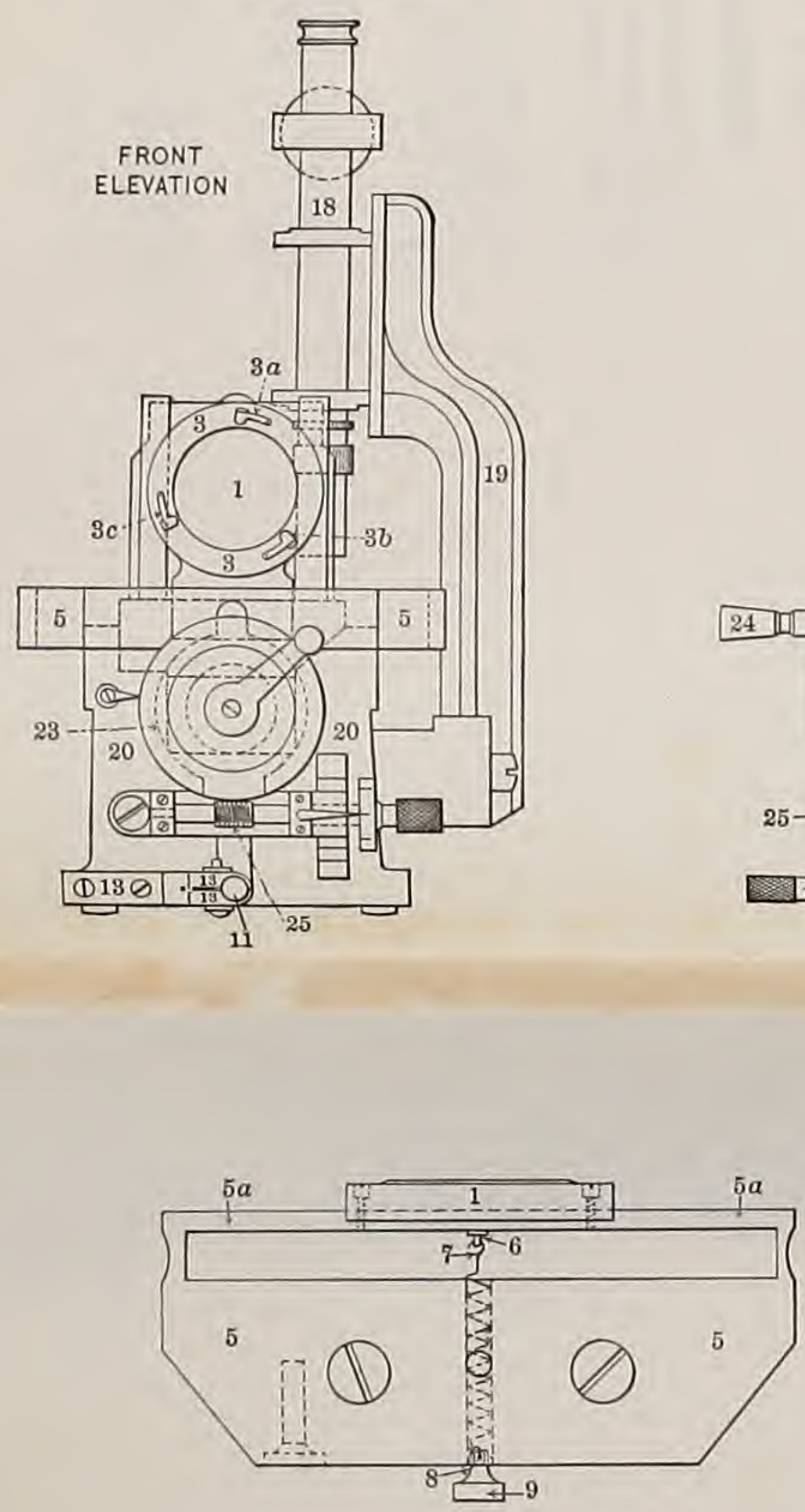

PLAN

Setting should not be much greater than $1 \mu$.
19. Bracket screwed to bed 20 and supporting 18 .
20. Bed of the interferometer in which are cut the

20. Bed of the interferometer in which are cut

22. Nut working on 21 .

22a. Pin projecting from 22 and working in slot in 15 .

23. Worm wheel on 21 .

24. Crank for turning 21

25. Tangent screw engaging 23.

26. Glass plate for reflecting light into the interferometer

FIG. 3
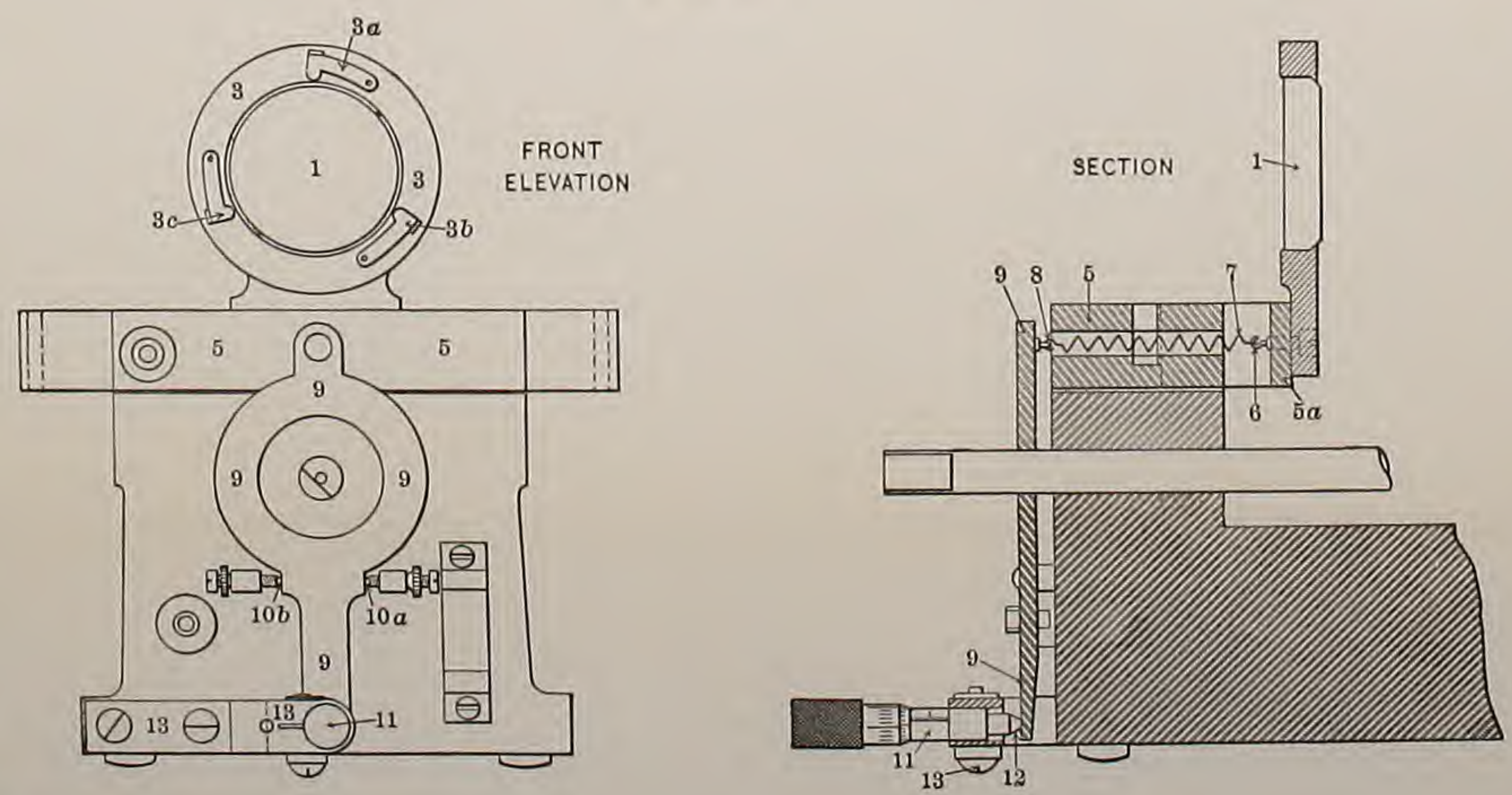


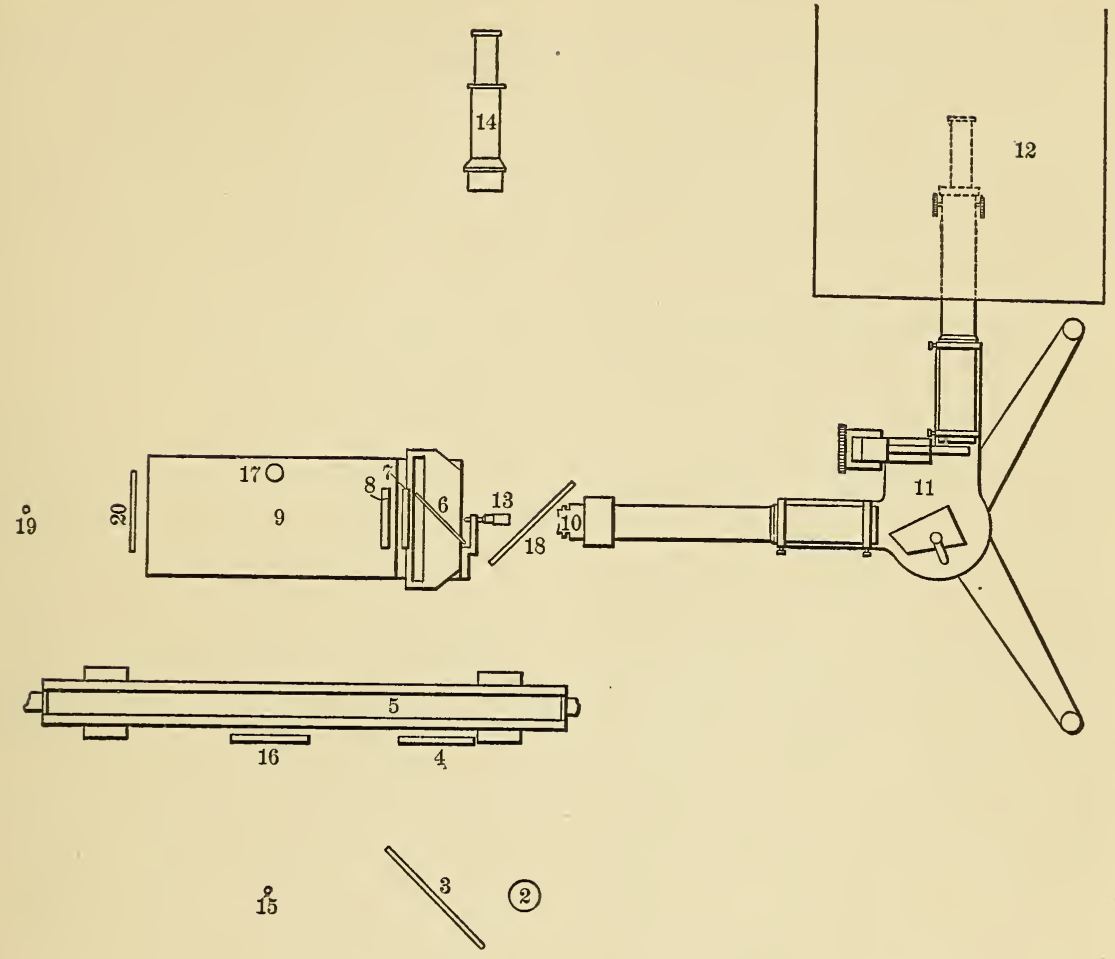

(1)

Fig. 4.-General plan of apparatus

KEY.

I. Source.

2. Source.

3. Transparent mirror for superposing two beams.

4. Condensing lens.

5. Water screen to protect interferometer from heat of lamps and heater.

6. Transparent mirror for reflecting light into the interferometer.

7. Partial interferometer mirror.

8. Full interferometer mirror.

9. Interferometer bed.

Io. Short focus (e. g., $20 \mathrm{~mm}$.) converging lens to form real image of interference pattern.

I I Spectrometer:
12. Dark observing hood over spectrometer ocular.

I3. Micrometer.

I4. Telescope used by assistant observer in reading micrometer.

15. Nernst lamp for illuminating scale on carriage.

r6. Condensing lens.

17. Microscope.

18. Removable mirror for observing the fringes, looking from the side.

19. Nernst lamp used in the preliminary adjustment of the parallelism of 7 and 8 .

20. Double convex lens. 
Microscope (I 8, Fig. 2), with micrometer ocular, by Zeiss. Magnification, about 25. Pitch of micrometer screw, $0.3 \mathrm{~mm}$. Smallest division on head (O.OI) corresponds to I $\mu$ on the scale. Lens (I o, Fig. 4), "Planar," by Zeiss. Focal length $=20 \mathrm{~mm}$. Spectrometer (I I, Fig. 4), Hilger's Constant Deviation type, with dense prism. ${ }^{15}$

\section{EXPERIMENTAL DETAILS AND PRECAUTIONS}

\section{PRELIMINARY ADJUSTMENTS}

The apparatus being arranged roughly, as shown in Fig. 4, the sources, I and 2, the partial mirrors, 3 and 6 , and the lens, 4 , must be so adjusted that the center of 7 , the image of the center of 4 in 6 and the images of the mid points of $\mathrm{I}$ and 2 in 6 shall be on a straight line normal to the plane of 7 . By sighting and measurements with a millimeter scale the several parts are brought into, roughly, their correct positions. A mirror, 18 , is placed between ro and 6 at a convenient angle, so that the image of 7 can be seen in it on looking from the side toward I4. The observer then moves his head until he sees the image of his observing eye centrally placed in 7 . He then turns 6 until the image of the frame of 4 has position concentric with the frame of 7 . Small movements of $I$ and 2 then serve to bring their distorted images central in the field. To make the first adjustment of the interferometer mirrors to approximate parallelism, a Nernst glower at the principal focus of a converging lens is placed on the opposite side of the interferometer from the spectroscope. The intense light of the glower suffices to penetrate even the heavy silver mirror in perceptible amount, so that in looking into 18 one sees a series of images of the glower due to multiple reflections between 7 and 8 . By adjusting the angular position of 8 these images are superposed. The observer, remaining in the same position, now views the interferometer in monochromatic light from I or 2, a piece of ground glass having been inserted between the source and the lens 4 . The interference fringes are now found and are made to assume the circular form by further angular adjustment of the position of 8 . The final adjustment of the parallelism is then made by adjusting the position of 8 until the fringes neither 
expand nor contract on moving the eye across the field in any direction. (There will usually be a small error in the plates that can not be corrected by altering their position.) The mirror, 18 , is now removed. The lens, ro, having been previously focused on the slit, and the telescope ocular on the image of the slit, the observer now goes to the spectroscope. By slightly turning the whole spectroscope the center of the image of the interference rings is brought upon the slit. By moving the whole spectroscope in direction perpendicular to the collimiator axis the position of best illumination is found. The rings and the slit are finally sharply focused by proper adjustments of the lens, Io, and the spectroscope ocular.

\section{COURSE OF A SINGLE DETERMINATION}

The preliminary adjustments being completed, the data for one determination are obtained and recorded as follows.

A. General data relative to the determination are recorded as shown in Form I. The numbers referring to apparatus are merely Bureau of Standards' inventory numbers, except the numbers of mirrors, which are serial numbers, as prepared. "Exper'l Value" is the arbitrary weight assigned the determination by the observer on account of experimental conditions. Only three numbers, 1,2 , and 3 , are used, 3 denoting best conditions and $\mathrm{I}$ the poorest conditions under which the determination would be given any weight at all.

B. Data for computing $\phi_{x 1}$ and $\phi_{r 1}$ are recorded as shown in Form 2. Columns headed $\mathrm{A}_{1}, \mathrm{~B}_{1}, \mathrm{C}_{1}, \mathrm{D}_{1}$ are the micrometer head readings for positions $a_{1}, b_{1}, c_{1}, d_{1}$ of the partial mirror as explained under $\mathrm{V}$. These numbers are read and recorded by an assistant observer as the observer at the spectroscope makes the corresponding settings by turning the micrometer screw. The eight numbers in each set (the sets being numbered I, II, III, etc.) are recorded in order across the page from left to right in the upper row of four and then back again from right to left in the lower row of four. The ten sets are obtained one after the other with as little delay as possible between; and any marked interruption is noted on the margin. 
C. Ten microscope readings on the scale for this position of the mirror are then obtained and recorded as shown in the left-hand column, Form 6. Temperature of scale is recorded.

D. After the carriage with the full silvered mirror has been displaced through the distance $\Delta t$ the data for computing $\phi_{x 2}$ and $\phi_{r 2}$, exactly similar to that for $\phi_{x 1}$ and $\phi_{r 1}$, are obtained and recorded as shown in Form 4.

$\mathrm{E}$. Ten microscope readings on the scale for this new position of the mirror are then obtained as shown in the right-hand column, Form 6.

\section{SPECIAL PRECAUTIONS}

Certain conditions are at our command, a proper choice of which will decrease the effect of instrumental defects and render our operations less liable to errors and mistakes. In this connection we take especial precautions as follows.

A. A well known and frequently used interval of the scale (I 7 , Fig. 2) is used to measure $\Delta t$. The intervals we use have been independently calibrated by microscopic comparison with the standards of this bureau and by interference methods so as to reduce the chance of a large mistake or blunder in the value of the interval. The interferential calibration was done with the scale in the position in which it is actually used on the carriage.

B. $\Delta t$ is made very nearly equal to a whole number of scale divisions and the micrometer head readings in $M_{1}$ and $M_{2}$ are thus made nearly equal. By so doing any error in $\Delta t$ due to error in screw value is made negligible.

C. All the fractions $\left(\phi_{x 1}, \phi_{x 2}, \phi_{r 1}, \phi_{r 2}\right)$ are chosen small. By so doing the effect of any small departure of the behavior of the elastic system from Hooke's law is reduced and the effect of errors in $D_{1}$ and $D_{2}$ may be made negligible in comparison to similar errors in $A_{1}, A_{2}, B_{1}, B_{2}, C_{1}$, and $C_{2}$.

D. $\phi_{x 2}$ is chosen nearly equal to $\phi_{x 1}$, and $\phi_{r_{2}}$ nearly equal to $\phi_{r 1}$. It is apparent how this condition will be effective in eliminating the effects of departures of the elastic system from the conditions of perfect elasticity, for if $\phi_{x 1}$ and $\phi_{r_{1}}$ are measured a little too large or too small, $\phi_{x_{2}}$ and $\phi_{r 2}$ will be affected to a similar degree in the same sense, and since we use only the differences $\left(\phi_{x_{2}}-\phi_{x_{1}}\right)$ and $\left(\phi_{r_{2}}-\phi_{r_{1}}\right)$ the errors will be eliminated. 


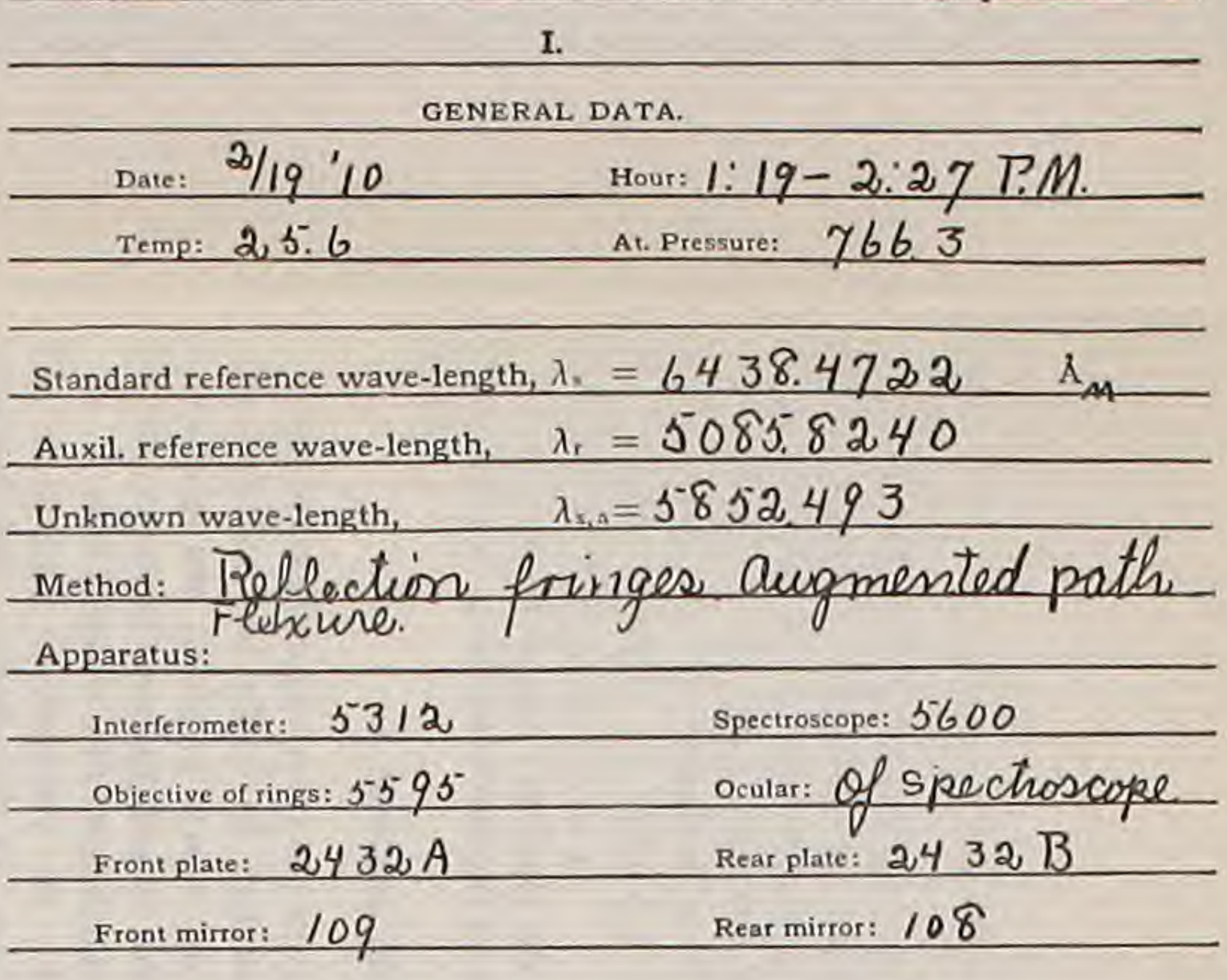

SPECIMEN RECORD FORMS ${ }^{16}$

\section{Forms 1 to 7 .}

${ }^{10} \mathrm{Not}$ all of the conditions mentioned under VII, 3 are perfeclly fulfilled in the specimen records bere given.

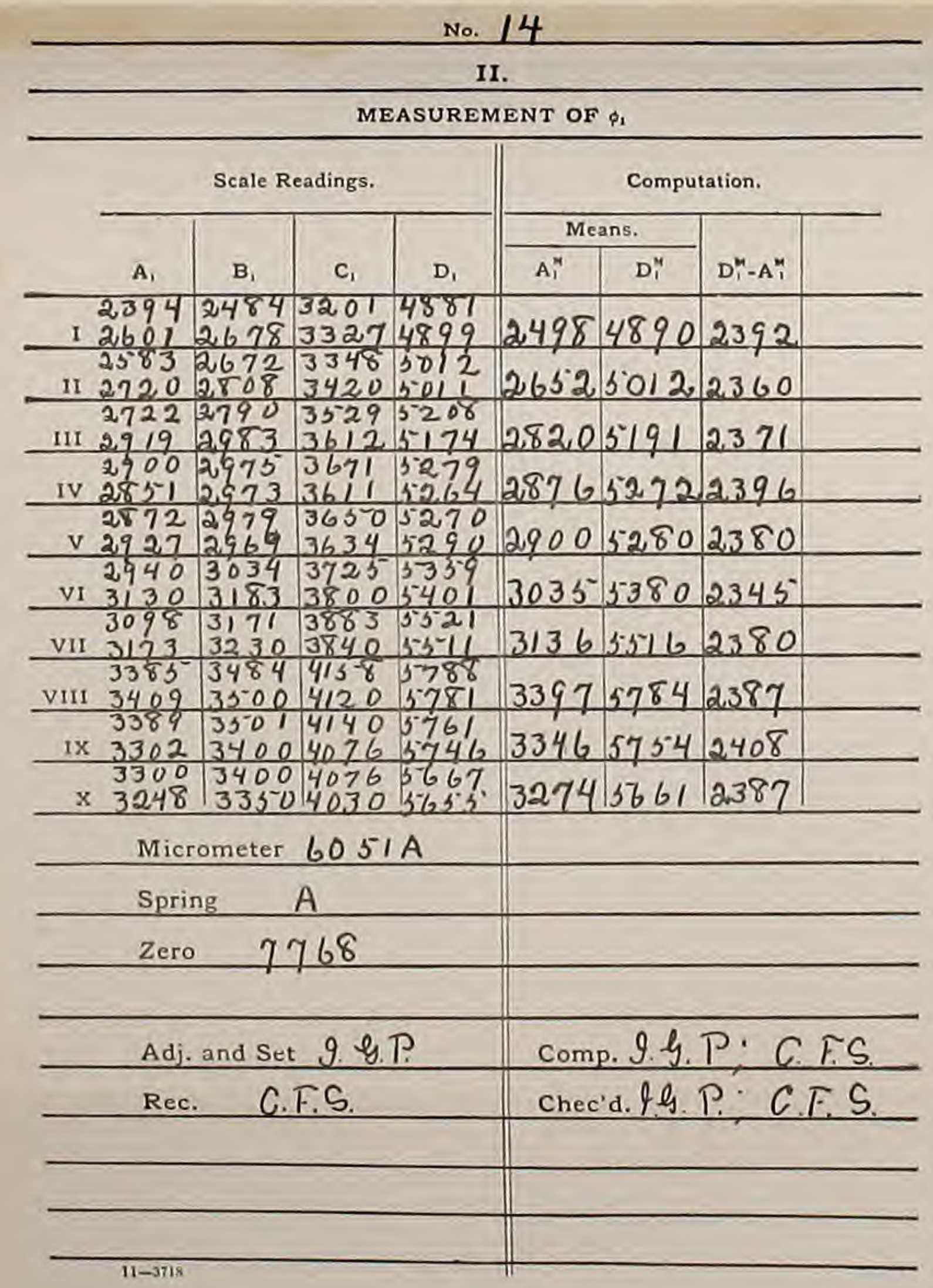

Form 2.

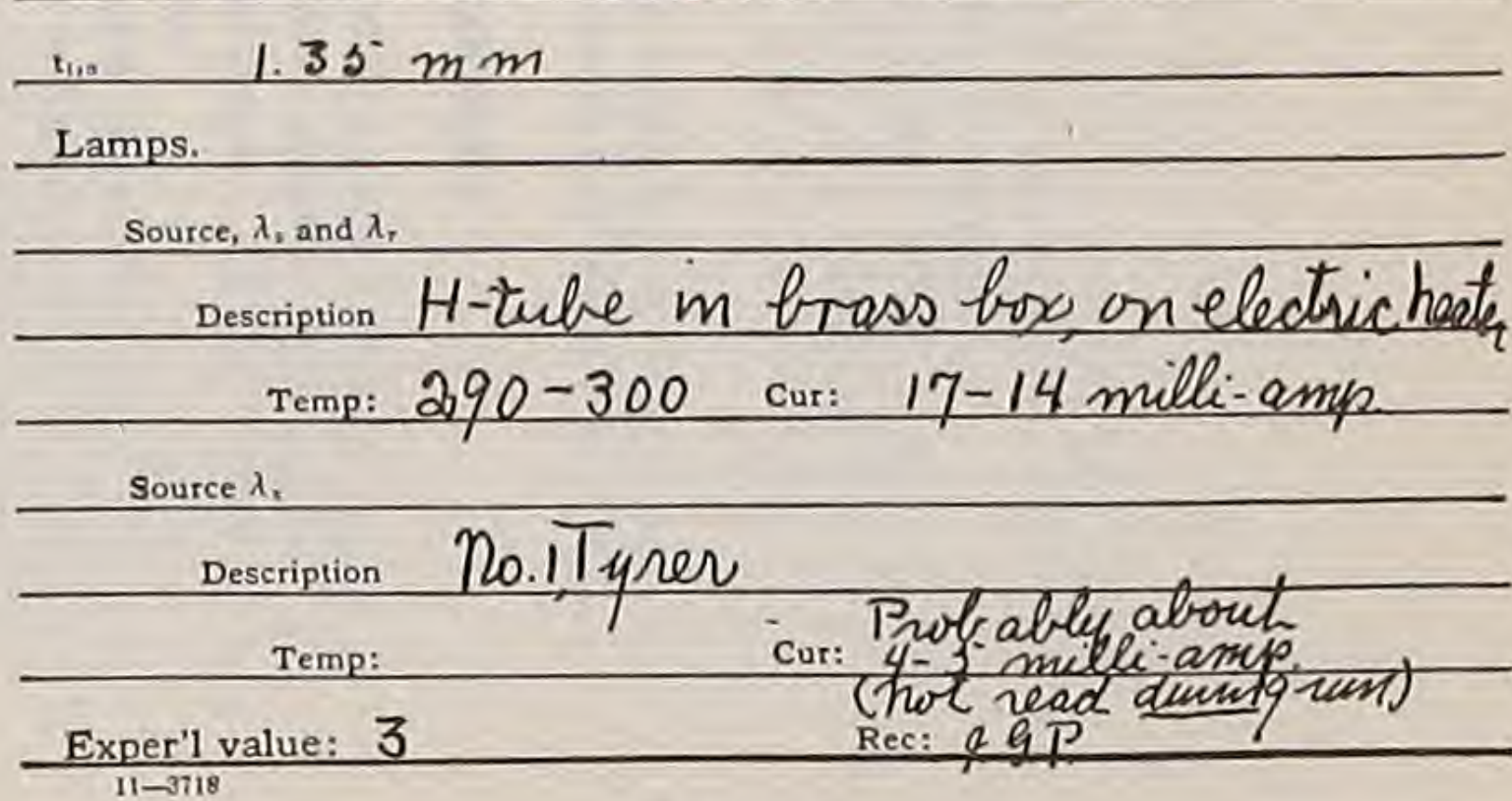

Form 1.

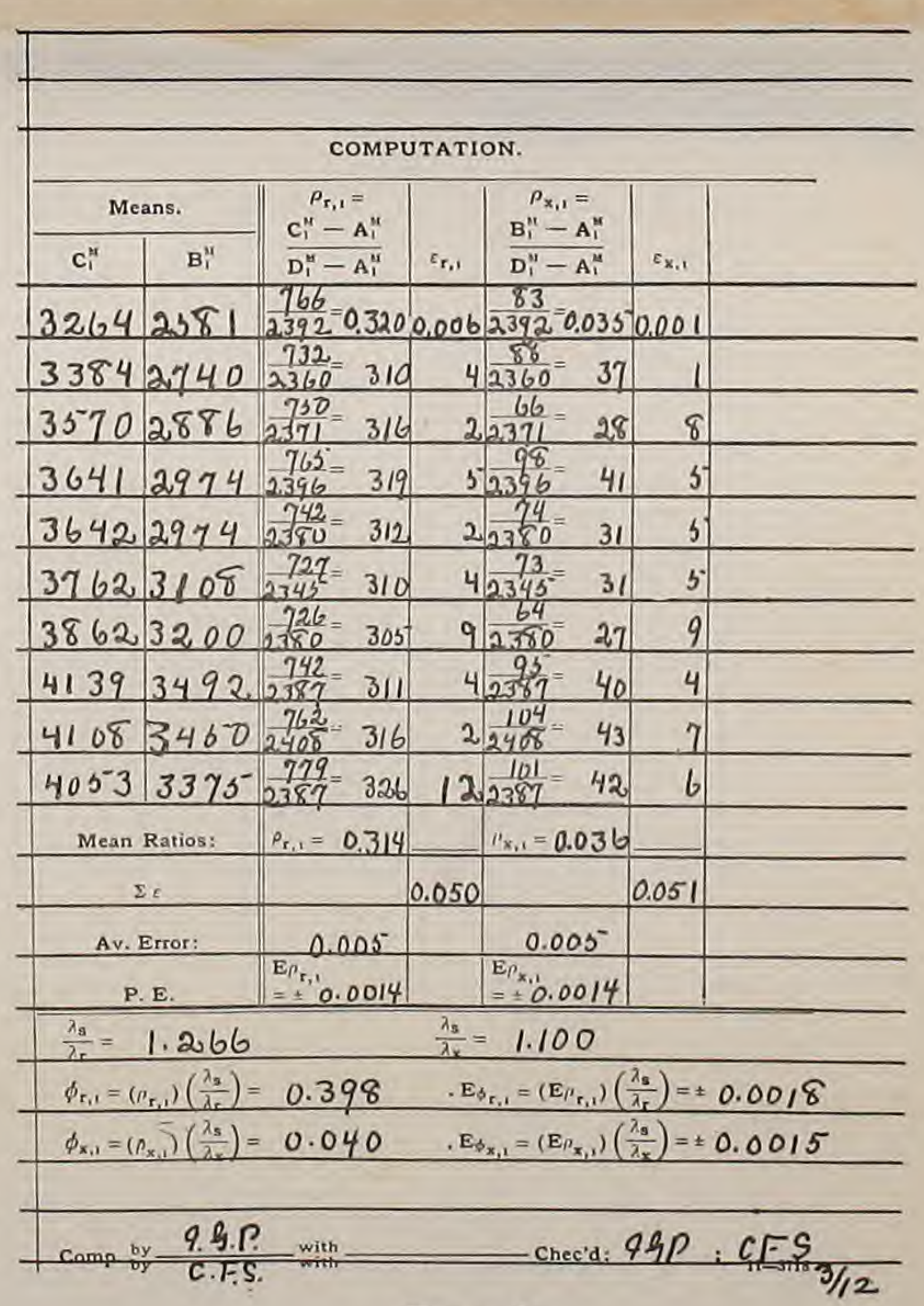

Form 3. 
No. 14

III.

MEASUREMENT OF $\phi_{y}$

Scale Readings.

\begin{tabular}{c|c|c|c|c|c|c}
$\mathrm{A}_{3}$ & $\mathrm{~B}_{1}$ & $\mathrm{C}_{2}$ & $\mathrm{D}_{2}$ & $\mathrm{~A}_{2}^{\prime \prime}$ & $\mathrm{D}_{2}^{\prime \prime}$ & $\mathrm{D}_{2}^{*}-\mathrm{A}_{2}^{*}$
\end{tabular} 2798318534405074267050652395

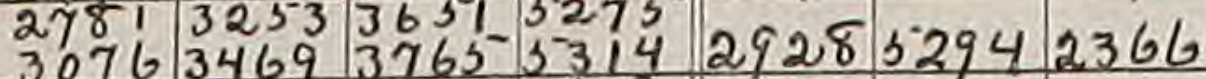
$\begin{array}{llll}3076 & 3469 & 3765 & 5314 \\ 3132 & 3570 & 3971 & 5629\end{array} 292942366$ 317236123931 3 331 3132, 38302478

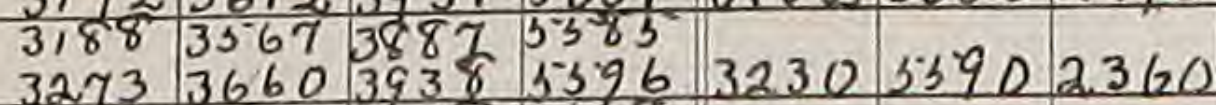
3231367140085628 3231
$v 3259$
3650
3897 $3925660 \quad 324537442399$

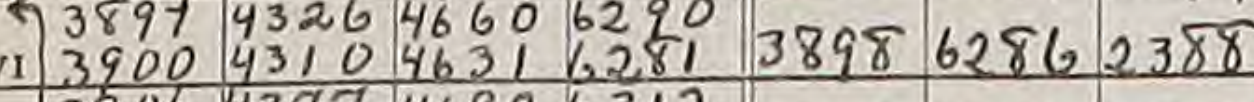
3906437746906312

$4021439846386281 \quad 396462962332$ 3984440247376323 III $3940433846036330 \quad 396263262364$ 3970432347326344 40044918016422408

$\begin{array}{r}40004491 \\ \times 4040444047966469402064642444 \\ \hline\end{array}$

Micrometer $6051 \mathrm{~A}$

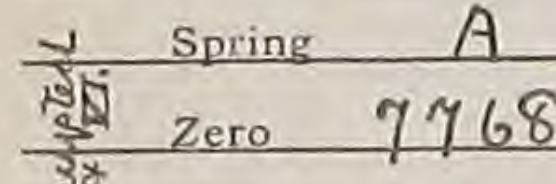

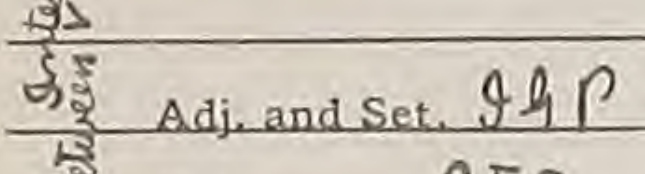
CFS comp. $q \& P-C F S$ chec'd. I \& $P$. CFS

Form 4.

No. 14

IV.

MEASUREMENT OF $\triangle t_{2}$

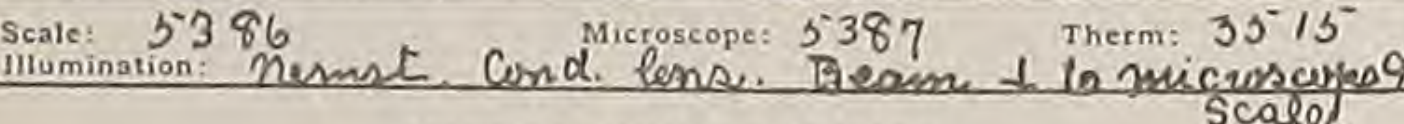
MICROSCOPE READINGS.

Before Displacement

Temp: $2.5 .5^{\circ} \mathrm{C}$

$1.435-23.0$

2.4

2.2

2.0

2.9

2. 6

2.1

2.2

2.2

1.8

\begin{tabular}{l|l}
$M_{1}=43522.34 \quad$ & $M_{2}=38021.24$
\end{tabular}

Av. Error: $\neq 0.084$

$K=\quad+3.8 \mu$

$\Delta t_{1}=M_{1}-M_{2}+K=5504.9$

$2 \Delta t_{a}=110098000$

17100

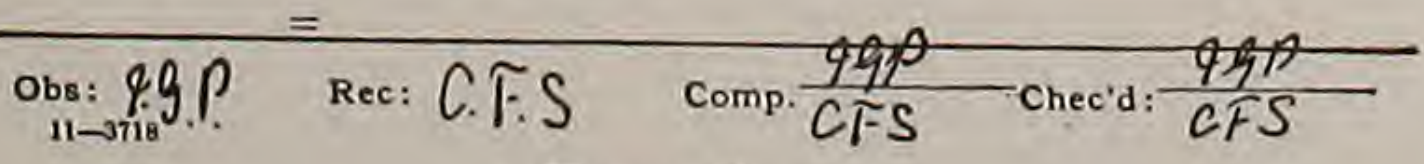
Form 6.

1.4
COMPUTATION.

\begin{tabular}{|c|c|c|c|c|c|c|}
\hline \multicolumn{2}{|c|}{ Means. } & \multirow{2}{*}{$\begin{array}{c}\rho_{\mathrm{r}, 2}= \\
\mathrm{C}_{2}^{\prime \prime}-\mathbf{A}_{2}^{\prime \prime} \\
\mathrm{D}_{2}^{\mathrm{H}}-\mathbf{A}_{1}^{\prime \prime}\end{array}$} & \multirow[b]{2}{*}{$c_{r, 2}$} & \multirow{2}{*}{$\begin{array}{c}\rho_{\mathrm{x}, 2}= \\
\mathbf{B}_{2}^{\mu \prime}-\mathbf{A}_{2}^{\prime \prime} \\
\frac{\mathbf{D}_{2}^{M \prime}-\mathbf{A}_{1}^{\mu}}{2}\end{array}$} & \multirow[b]{2}{*}{$E_{x, 2}$} & \\
\hline $\mathrm{C}_{1}^{\pi}$ & $B_{2}^{\prime \prime}$ & & & & & \\
\hline 3385 & 3083. & $\frac{713^{\circ}}{2395}=0.298$ & 0.012 & $\frac{412}{23,55}=0172$ & 0.002 & \\
\hline 3708 & 3361 & $\frac{780}{2366}=330$ & & $\frac{433}{2366}=183$ & 9 & \\
\hline 3951 & 3391 & $\frac{799}{2478}=322$ & & $\frac{439}{2478}=177$ & 3. & \\
\hline 3912 & 3614 & $\frac{682}{2360}=289$ & & $\frac{384}{2360}=163$ & 11 & \\
\hline 4000 & 3660 & $\frac{753}{2399}=315$ & & $\frac{415^{5}}{1399}=173$ & 1 & \\
\hline 4646 & 4318 & $\frac{74 \sqrt{8}}{2388}=313$ & & $\frac{420}{2388}=176$ & 2 & \\
\hline 4664 & 4388 & $\begin{array}{r}700 \\
2332\end{array}$ & & $\frac{424}{2332}=182$ & 8 & \\
\hline 4680 & 4370 & $\frac{718}{2364}=304$ & & $\frac{408}{2.364}=173$ & 1 & \\
\hline 4719 & 4343 & $\frac{753}{2405}=314$ & & $\frac{37 q}{2408}=157$ & 17 & \\
\hline 4798 & 4466 & $\begin{array}{ll}778 & 38 \\
2444 & 318\end{array}$ & & $\frac{446}{2444}=182$ & 8 & \\
\hline Mean & Ratios: & $\rho_{r, 2}=0.310$ & & $n_{x, 2}=0.174$ & & \\
\hline & 8 & & 0.101 & & 0.062 & \\
\hline $\mathrm{A} v_{2}$ & Error: & 0.010 & & 0.0062 & & \\
\hline & E. & $\begin{array}{l}E \rho_{r, 3} \\
= \pm 0.0028\end{array}$ & & $\begin{array}{l}E \rho_{x, 2} \\
=\quad 0.0017\end{array}$ & & \\
\hline$\frac{\lambda_{s}}{\lambda_{r}}=$ & 1.266 & & $\frac{\lambda_{3}}{\lambda_{2}}=$ & 1.100 & & \\
\hline$\phi_{n, 2}=(1$ & $\left.r_{r, 1}\right)\left(\frac{\lambda_{g}}{\lambda_{r}}\right)=$ & 0.392 & & $E_{u_{r, 2}}=\left(E_{r_{r, 2}}\right)$ & $\left(\frac{\lambda_{s}}{\lambda_{s}}\right)= \pm$ & 0.0035 \\
\hline$\phi_{x, a}=(1$ & $(x, 3)\left(\frac{\lambda_{5}}{\lambda_{x}}\right)=$ & 0.191 & & $E_{s_{x, 2}}=\left(E_{\rho_{x, 2}}\right)$ & $\left(\frac{\lambda_{s}}{\lambda_{x}}\right)= \pm$ & $=0.0019$ \\
\hline
\end{tabular}

$98 \mathrm{p}$

$44 P: C F S$

Form 5 .

No. 14

V.

COMPUTATION.

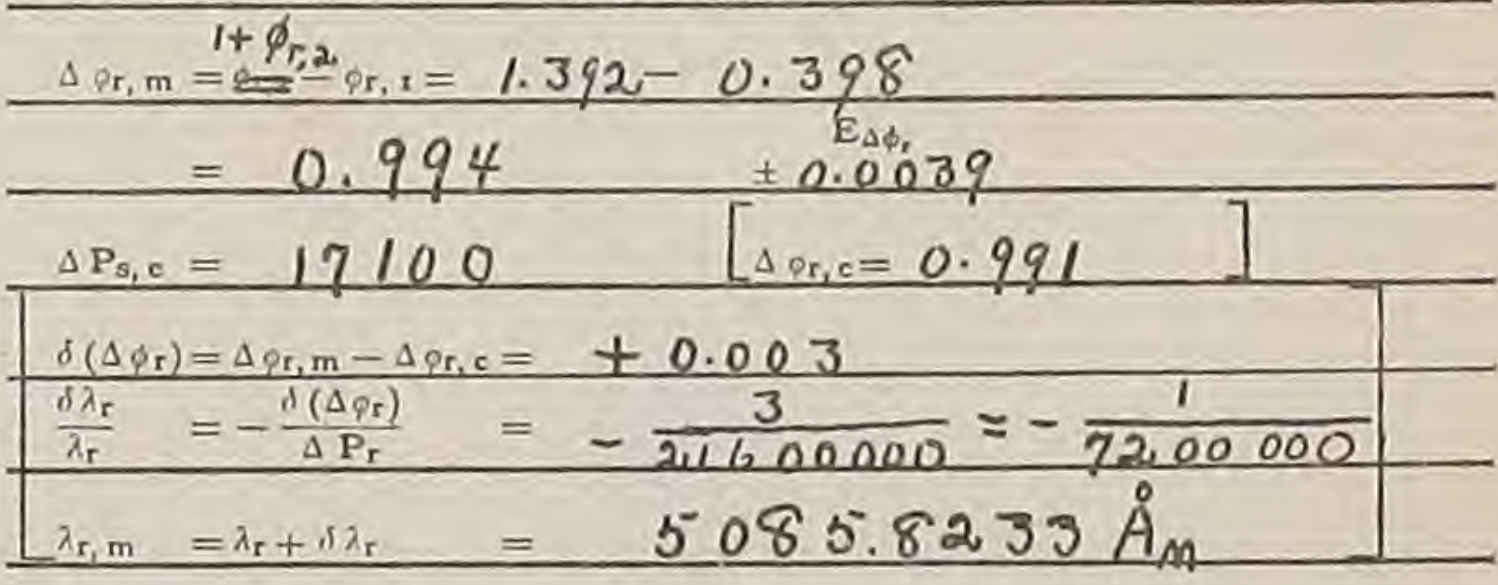

$2 \Delta t_{e}=\left(\Delta P_{s, e}\right) \lambda_{s}$

$\begin{aligned} & =110097875 \\ \Delta p_{x, a} & =\frac{2 \Delta t_{0}}{\lambda_{x, a}} \\ & =18812133 \\ \Delta \varphi_{x} & =\varphi_{x, 1}-\varphi_{x, 1}=0.191-0.040 \\ & =\quad 0.151\end{aligned}$

$\begin{array}{ll}\Delta P_{x, e}= & 18812.151 \\ \lambda_{x, e}=\frac{2 \Delta t_{e}}{\Delta P_{x, e}}\end{array}$

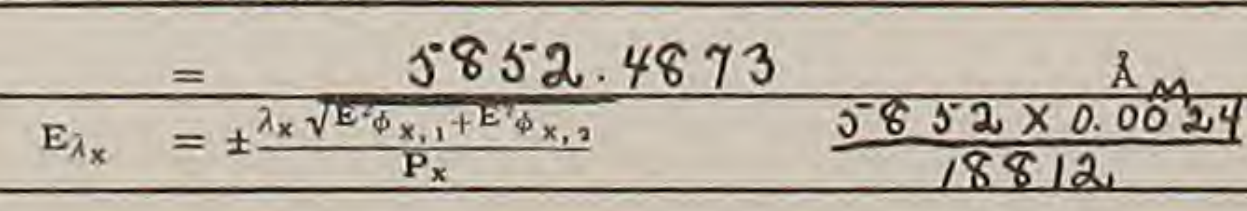

$= \pm 0.00074$

FINAL RESULT.

$\lambda_{x}$ ( Tably, Table, 5852.65

$5852.487 \pm 0.00074 A_{A}$

Comp. by $\frac{9 g P \text { with }}{\text { CFS with }}$ Chec'd: $9 q P$; CFS

$11-3718$

Form 7.

$4884^{\circ}-10$. (To precede page 597 .) 
E. $\overline{a_{1} b_{1}}$ is chosen nearly equal to but a little less than $\overline{a_{1} c_{1}}$, and $\overline{a_{2} b_{2}}$ nearly equal to but a little less than $\overline{a_{2} c_{2}}$. Since we can calculate, a priori, $\Delta \phi_{r}$ to a better accuracy than the precision attainable in the measured value $\left(\Delta \phi_{r m}=\phi_{r_{2}}-\phi_{r_{1}}\right)$, the degree of agreement between this calculated value $\Delta \phi_{r c}$ and the measured value $\Delta \phi_{r m}$ affords us a very valuable indication of the reliability of the measured fraction $\Delta \phi_{x}$, for, by the arrangement just mentioned, $\Delta \phi_{x}$ and $\Delta \phi_{r}$ are measured under almost identical conditions, such that the instrumental error should be nearly the same in both, but, if anything, greater in $\Delta \phi_{r}$ than in $\Delta \phi_{x}$; and we have obtained very closely the true value of the actual error in $\Delta \phi_{r}$.

Conditions $\mathrm{A}$ and $\mathrm{B}$ are satisfied by bringing the carriage into position while observing the scale through the microscope. Conditions $\mathrm{C}, \mathrm{D}$, and $\mathrm{E}$ can then generally be satisfied by very small displacements of the carriage while observing the fringes through the spectroscope and without disturbing conditions $A$ and $B$ to an undesirable extent.

\section{COMPUTATION}

The course of the computation is shown in Forms 2, 3, 4, 5, 6, and 7 . Forms 2 and 3 show the computation of $\phi_{r_{1}}$ and $\phi_{x_{1}}$. Forms 4 and 5 show the computation of $\phi_{r_{2}}$ and $\phi_{x_{2}}$. Form 6 shows the computation of $\Delta t_{a}$ and $\Delta P_{s a}$. Form 7 shows the determination of $\Delta P_{s e}$ and $\Delta p_{x e}$, and the final computation of the relative wave-length.

\section{TESTS OF PRECISION AND RELIABILITY}

\section{DEFINITION OF TERMS}

By precision we mean the degree of agreement of individual measurements or determinations. As a convenient measure of the precision of the result of a single determination we take its probable error as computed above from the residuals in the ro measurements of $\phi_{1}$ and Io measurements of $\phi_{2}$. When a number of determinations are available, as in the examples below, a further indication of the precision of the method is afforded by the differences between individual results and their mean. By reliability we mean the degree to which the method may be depended upon to give the correct result within the limits of uncertainty fixed by the precision. 


\section{PRECISION}

The magnitude of the irremovable accidental errors is fixed by the acuity of the observer's judgment in estimating the positions for disappearance of the bright centers. In our present apparatus, we probably have superposed upon this the effect of irregularities in the action of the flexure apparatus. The accidental errors due to both of these causes appear in the probable errors of $\phi$ computed as shown in the forms mentioned above. Table I contains a résumé of probable errors of $\phi$ computed as above for different conditions indicated in the table, $\lambda_{s}$ being in all cases cadmium 644 .

\section{TABLE I}

$E_{\phi}$

\begin{tabular}{|c|c|c|c|c|c|c|c|}
\hline & & \multicolumn{2}{|c|}{ Cd 509} & \multicolumn{2}{|c|}{ Ne 585} & \multicolumn{2}{|c|}{ Ne 594} \\
\hline \multicolumn{2}{|c|}{$p a$} & 5300 & 26900 & 4600 & 23400 & 4500 & 23000 \\
\hline \multirow{6}{*}{ 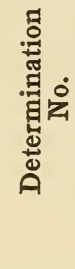 } & 12 & & & 0.0012 & 0.0019 & & \\
\hline & 13 & 0.0012 & 0.0017 & 8 & 16 & & \\
\hline & 14 & 18 & 35 & 15 & 19 & & \\
\hline & 18 & & & 14 & 22 & 0.0009 & 0.0014 \\
\hline & 19 & & & 13 & 15 & 10 & 18 \\
\hline & Mean & 0.0015 & 0.0026 & 0.0012 & 0.0018 & 0.0010 & 0.0016 \\
\hline
\end{tabular}

Noting that the probable error of $\Delta p$ is given by $\pm \sqrt{E_{\phi_{1}}^{2}+E_{\phi 2}^{2}}$ and taking $\Delta p$ as the difference of the two values of $p_{a}$ under each wave-length; and $\phi_{1}$ and $\phi_{2}$ respectively as the values of $\phi$ under the small and large values of $p_{a}$, it follows from this table that, for these radiations and these values of $p$, the average probable error of $\Delta p$ is about one part in 8000 ooo. From which we have also: the average probable error of $\lambda$ for the best lines is about one part in 8000 ooo.

Further evidence relative to the precision we have attained in the use of this method is given in Table II. 


\section{TABLE II}

Determination Relative to Cd Standard, of Neon Line, $\lambda_{x a}=5852.65^{17}$

\begin{tabular}{|c|c|c|c|c|c|}
\hline $\begin{array}{c}\text { Determination } \\
\text { No. }\end{array}$ & Date & $\Delta p$ & $\stackrel{\lambda}{\lambda}$ as determined & $E_{\lambda}$ & Residuals \\
\hline 11 & $2 / 5, ' 10$ & 18811 & $\begin{array}{c}\AA_{\mathrm{M}} \\
5852.4888\end{array}$ & $\AA$ & $\begin{array}{c}\AA \\
0.0002\end{array}$ \\
\hline 12 & $2 / 15$ & 18822 & 876 & \pm 0.00070 & 0.0010 \\
\hline 13 & $2 / 15$ & 18790 & 884 & 56 & 2 \\
\hline 14 & $2 / 19$ & 18812 & 873 & 74 & 13 \\
\hline 16 & $3 / 1$ & 35919 & 889 & 47 & 3 \\
\hline 18 & $3 / 1$ & 18734 & $901^{\circ}$ & 81 & 15 \\
\hline 19 & $3 / 3$ & 18801 & 882 & 62 & 4 \\
\hline Means & & & 5852.4886 & 0.00065 & 0.00070 \\
\hline
\end{tabular}

17 Baly's Table relative to Kayser's Iron Standards. Trans Roy. Soc., A, 202, p. I83. Proc. Roy. Soc., 72, p. 84; 1903 B. A. Report; 1905, pp. 105-108.

\section{REMARKS ON TABLE}

The above table contains every determination I have made of this wave-length relative to $\mathrm{Cd} 6438.4722$ with values of $\Delta p$ large enough to give this order of accuracy. Preliminary determinations with low orders of interference gave values of from $585^{2.480}$ to $5^{8} 5^{2.497}$, relative to $\mathrm{Cd}, 6438.4722$ and $\mathrm{Hg}, 5460.7424$. These preliminary approximate values were used as already explained in obtaining the more accurate values given above. No. I I is based on only 5 measures each of $\phi_{1}$ and $\phi_{2}$. All the other determinations are based on ro measures of each of these fractions. In determinations 18 and 19 , this wave-length functioned as $\lambda_{r}$ instead of $\lambda_{x}$, but as its value is already known within certain limits, the data obtained in these determinations enable us to compute an independent value. No. 12 is subject to slight suspicion.

The following are the important conclusions from the table.

The average probable error of the last six determinations is about one part in 9 ooo ooo.

The average residual of the seven determinations is about one part in 8400 ooo.

The maximum residual of the seven determinations is about one part in 3900000 .

The foregoing data are thought to be sufficient for forming an estimate of the precision attainable in measuring the wave-lengths of the best lines. 


\section{RELIABILITY}

The reliability of a method of measurement depends upon the correctness of the theoretical relations assumed in formulating the method and upon the degree to which the actual experimental procedure satisfies the ideal conditions of the theory. Errors of method are of two classes. It may be that the experimental procedure is such as to always fail, to the same degree and in the same sense, to satisfy the relations on which the method is based. Such a condition will cause a constant error. Or it may be that the experimental result is a function of some variable condition (e. g., temperature or pressure) not recognized in formulating the method. Variation of this condition may cause in one individual determination a positive error and in another a negative error; but unless such errors are small and are, in any set of determinations, likely to be positive as often as negative they can not generally be satisfactorily eliminated by multiplying determinations.

Ordinarily the discussion of the reliability of a method involves a searching investigation of all conceivable sources of error, and we can never be certain that some have not escaped consideration. The obvious sources of errors and mistakes and the procedure for eliminating them have been noted under VII, 3. Fortunately, in the present instance we are relieved of the necessity of making an exhaustive investigation of errors of method because of the possibility of making control determinations. The ratio of the wave-lengths of the green and red radiations of cadmium is known within an uncertainty of only about one part in 20000000 from Michelson's work on the meter. The most rigorous test that can be applied to a method for relative wave-lengths is the ability to check this ratio to the accuracy desired in the method under test. One such test would give us an indication of the importance of the first class of errors of method mentioned under "Reliability." It would not, however, relieve us of concern as to the second class. The highest degree of assurance in this regard can only be obtained by running a control determination in connection with every determination of an unknown, and arranging the experiment in such a way that the chance of errors affecting the determination of the unknown and not affecting the control will be minimized and further arranging so that, as far as we can judge 
a priori, the error of method in the control will, if anything, tend to be larger than in the determination of the unknown. An examination of our Record Forms 2, 4, and 7 and consideration of the precaution mentioned under VII, $3, \mathrm{E}$, will show that the determination of $\Delta \phi_{r m}$ affords the data for such a control determination closely interwoven with the determination of the unknown. The identification of $\Delta P_{s e}$, which is the primary use to which $\Delta \phi_{r m}$ is put, does not require a very accurate value of this fraction. However, by obtaining a value of the same order of precision as $\Delta \phi_{x}$, we have a rigorous check on the reliability of $\Delta \phi_{x}$. Definitely, we have

$$
\frac{\Delta p_{r m}-\Delta p_{r c}}{\Delta p_{r}}=-\frac{\delta \lambda_{r}}{\lambda_{r}}
$$

where $\delta \lambda_{r}$ is the error in $\lambda_{r}$ due to using $\Delta p_{r m}$ for $\Delta p_{r c}$. We may safely assume, roughly (say to an approximation of one part in ten), that

$$
\frac{\delta \lambda_{x}}{\lambda_{x}}=\frac{\delta \lambda_{r}}{\lambda_{r}}
$$

where $\delta \lambda_{x}$ is error of method in $\lambda_{x}$

Control determinations we have made give actual departures from Michelson's value of the ratio of the red and green cadmium wave-lengths as shown in Table III.

TABLE III

\begin{tabular}{c|c}
\hline Number of determinations of $\Delta \phi_{r}$ & Departure \\
\hline 10 & $\frac{1}{7200000}$ \\
10 & $\frac{1}{21600000}$ \\
5 & $\frac{1}{3100000}$ \\
10 & $\frac{1}{2300000}$ \\
\hline
\end{tabular}

It should be explained that the third and fourth of these values were obtained under unfavorable conditions, which would now be avoided in careful work. Also it should be noted that the third 
is based on only five measurements of $\Delta \phi$. This evidence is suffcient to show that the systematic error can be kept well within the accidental error. Of course when the wave-lengths of other suitable radiations are well enough known they may be used as controls instead of cadmium green.

The only errors that occur to me as not being detected by the above procedure are those that might be due to peculiarities of the radiation $\lambda_{x}$ not shared by the radiation $\lambda_{r}$. Such peculiarities may occur in brightness, hue, and "line structure." An examination of the method will show that a constant difference in brightness between $\lambda_{x}$ and $\lambda_{s}$ will not introduce an error, but that a change in brightness of either between the measurements of $\phi_{1}$ and $\phi_{2}$ might be expected to do so. However, a simple direct experiment shows that even doubling the current through our Neon lamp does not sensibly change the adjustment for disappearance of the bright center. It will be well to guard against large variations in the intensities of illumination in the field, but no excessive or troublesome precautions need be taken on this account. As to errors due to hue differences, the cadmium green radiation is most favorably situated with respect to red to make this error large. As this is our control we need hardly fear that the errors for other wave-lengths would be greater. As to the errors due to "line structure" it may be said that the presence of satelites may vitiate the measurements. We do not ordinarily obtain correct results in using mercury 546I as a working standard. As, however, such lines should not be chosen as standards, ${ }^{18}$ the difficulties in this connection need not trouble us. Apparently the green cadmium satelite has not troubled us, probably because of its faintness. A change in the width or reversal of a line between the measurement of $\phi_{1}$ and $\phi_{2}$ would no doubt introduce an error. On this account the excitation of the source must be kept constant within limits that may be fixed by previous knowledge of the behavior of the line with changing excitation.

Additional assurance as to the reliability of the method is afforded by the close agreement obtained between the value of $\Delta t$ measured with the microscope and scale and the more accurate value corresponding to $\Delta P_{s e}$. In the seven determinations noted

${ }^{18}$ Fabry and Buisson were able to use this line as a working standard, taking the precaution to obtain its relation to the primary standard for each pair of mirrors used. 
above, these two values always agreed to within a few tenths of a micron, always to within less than one wave-length. For the purpose of determining $\Delta P_{s e}$ by the method used, the microscopic determination of $\Delta t$ need not be of this degree of accuracy, but it is highly satisfactory to have the agreement so good. One feels that he is working with a larger "factor of safety," the chance of blundering in identifying $\Delta P_{s e}$ being minimized.

Any conclusions as to the precision and reliability of the method drawn from this paper are of course limited by the extent of the data here presented. It has been shown that in the case of one particular ratio, results of a high degree of precision can be obtained. It has been shown that the method checks, to a high degree of accuracy, the previously known ratio of the red and green cadmium wave-lengths, and a plan for running a rigorous control on each determination has been formulated and presented. Probable trouble with lines of complex structure and variable width has been pointed out. There is no apparent reason why the method should not give, for other lines well suited to serve as standards, results comparable in precision and reliability with those given above, provided proper arrangements for observation can be made.

It is expected that the method will be more extensively tested at this laboratory during the coming year. The author would suggest the desirability of independent tests being undertaken by other observers in other laboratories at the same time.

\section{ADVANTAGES OF THE METHOD}

This method seems to the author to possess the following advantages over the method of diameters as now universally used in establishing spectroscopic standards.

I. The procedure is more direct, involves fewer assumptions, and permits of a more rigorous control of the errors.

2. There is less chance of apparatus errors, such as are due to imperfections of the mirrors, errors in adjustments, and aberations of the optical system, the requirements of instrumental perfection being much less severe, thus giving a considerable "factor of safety."

3. The chance of error due to distortion of the photographic image is eliminated. By many this source of error is not consid- 
ered serious. However, Albrecht ${ }^{19}$ has shown the existence of photographic distortion that must be considered if an accuracy of one part in 4000000 is to be obtained in all cases with the method of diameters as now used.

4. The errors due to small temperature changes are eliminated without resorting to a thermostat.

5. The error due to "dispersion of phase" on reflection is automatically eliminated without further discussion or correction. Some idea of the importance and trouble of this correction may be obtained by merely noting that in Eversheim's recent paper on the iron standards Io pages out of a total of 24 are taken in its discussion.

6. The personal error in the measurement of the order of interference is eliminated to a greater extent than in the method of diameters. It is to be noted that in the method of diameters the wave-length is derived from the measurement of the diameter of a circle. It is obvious that this condition is very different from measuring a length defined by two straight lines and is favorable to the introduction of a personal equation. Data presented by Plund ${ }^{20}$ shows that his personal error in this measurement whatever its size is not constant from day to day. It is true that this error would be eliminated if the diameters of the rings for the standard and the unknown wave-lengths were equal. But this condition will not in general be satisfied; so that complete compensation will be only accidental.

7. There are fewer chances for accidental error because while in the method of diameters the precision of each determination is sensibly affected by the accidental errors of at least eight scale readings, ${ }^{21}$ the precision of each determination by the present method is affected by the accidental errors of only six scale readings, and by two of these in so slight a degree as to make the precision of the measured wave-length sensibly a function of the errors of only four scale readings.

${ }^{19}$ Astrop. Jour. 25, p. 349; I907.

${ }^{20}$ Astrop. Jour. 28, p. 210 ; 1908.

${ }^{21}$ In all, I 4 or 18 scale readings plus the number or readings required for a determination of focal-length are involved in each wave-length determination, but the errors of some of them are small enough to be negligible in comparison with the others. 
Data available are not adequate for making a rigorous or very significant comparison of the degrees of precision attained in the two methods. I have tried to estimate the relative precision by comparing the probable errors published by Eversheim ${ }^{22}$ with my own probable errors given above. Unfortunately, a direct comparison is not significant for the following reasons: (I) Eversheim's method of computing his probable errors is not sufficiently specified; (2) apparently his published probable errors do not take into account all the accidental errors to which his method is subject, ${ }^{23}$ while mine do include all accidental errors in the present method; (3) the individual wave-length values in his table result from 36 measurements of the order of interference, while the individual values given by me result from only ro measurements; (4) our data are for different radiations. It is hoped that data sufficient for a significant comparison of the degrees of precision of the two methods will be available in a few months and a definite comparison in this respect is therefore not attempted now.

\section{CONCLUSION}

The result of this investigation has been the definite formulation of a method which it is believed will give a closer approximation than has hitherto been attained to the ideal of an uncertainty less than I part in 4000000 . Conclusions as to the precision and reliability of the method have been formulated from experiment and stated above (pp. 599,602,603) and the peculiar advantages of the method have been pointed out (X). It is not thought that the results above presented are the best of which the method is capable. Improvements under way or being considered are: (I) Improvement of the flexure apparatus by substituting a cut-steel spring for the wound-wire spring and perhaps the substitution of a crystalline-quartz bar for the steel bar; (2) substitution of a black and white micrometer head that can be more easily and rapidly read than the present one (II, Fig. 2); (3) better achromatism of the lenses in the observing apparatus; (4) a slight

\footnotetext{
${ }^{22}$ Ann. der Phys. 30, pp. 837, 838; November 30, I909.

${ }^{23}$ Eversheim's probable errors are evidently intended merely to indicate the relative precision of his different measurements and not the precision of the method.
} 
increase in $\Delta p$. It should also be noted that in Table II the determinations having the large probable errors actually give the largest residuals. It is hoped in future determinations to keep the probable errors considerably smaller than these largest ones.

We are now using the method to determine about 20 wavelengths in the red, orange, and yellow of the neon spectrum. These lines are exceedingly well suited to serve as standards on account of their homogeneity, their brightness, and the convenience of maintaining the source. Being in a region of the spectrum where iron lines are unsatisfactory, it is thought their accurate values will be of considerable service to spectroscopists. It is hoped to publish a table of these wave-lengths within a few months. WASHINGTON, June, I9IO.

NoTE.-A photographic method for recording the micrometer-head readings (see VII, 2, B above) is now in use. Besides eliminating the necessity of an assistant observer this modification promises to aftord an improvement in the method.

DECEMBER I 5, I9IO. 